\title{
Reactive Matrix-Assisted Laser Desorption/lonization Mass Spectrometry Imaging Using an Intrinsically Photoreactive Paternò- Büchi Matrix for Double-Bond Localization in Isomeric Phospholipids
}

\author{
Fabian Wäldchen ${ }^{\mathrm{a}}$, Bernhard Spengler ${ }^{\mathrm{a}}$ and Sven Heiles ${ }^{\mathrm{a} *}$ \\ ${ }^{a}$ Institute of Inorganic and Analytical Chemistry, Analytical Chemistry, Justus Liebig University \\ Giessen, 35392 Giessen, Germany
}

\section{Supporting Information}

\author{
*Address correspondence to Dr. Sven Heiles: \\ Institute of Inorganic and Analytical Chemistry \\ Justus Liebig University Giessen \\ Heinrich Buff Ring 17 \\ 35392 Giessen, Germany \\ Phone: +49 6419934807 \\ e-mail: sven.heiles@anorg.chemie.uni-giessen.de
}




\section{Materials}

Lipid standards and sphingomyelin brain extract (porcine) were purchased from Avanti Polar Lipids (Alabama, USA) and NOF Corporation (New York, USA). All other chemicals (analytical grade or higher) were obtained from Sigma-Aldrich and used without further purification.

\section{Lipid nomenclature}

The lipid nomenclature introduced by Liebisch et al. ${ }^{1}$ is used except for DB-position notations for which the $n$-nomenclature is employed. ${ }^{2}$ Consequently, a fatty acyl with $18 \mathrm{C}$-atoms and one DB at the 7th $\mathrm{C}$-atom counted from the aliphatic end of the hydrocarbon residue, is FA 18:1 (n-7).

\section{Tissue and sample preparation}

Mouse brain (C57BL6/N male and female mice 12-20 weeks of age) samples were cut into sections of $20 \mu \mathrm{m}$ thickness with a cryotome (HM525, Thermo Scientific, Dreieich, Germany) at $-20^{\circ} \mathrm{C}$, thaw-mounted on glass slides and stored at $-80^{\circ} \mathrm{C}$ until sample preparation. Tissue sections were washed with a $150 \mathrm{mmol} / \mathrm{L}$ ammonium formate-solution before matrix application to remove alkali metal ions and promote formation of protonated ions. Intact matingexperienced male S. mansoni worms (from Mesocricetus auratus hosts as described in ref. ${ }^{3}$ ) were stored at $-80{ }^{\circ} \mathrm{C}$ in $150 \mathrm{mmol} / \mathrm{L}$ ammonium formate-solution until use. Before matrix application, the worms were washed with water. For the application of benzophenone (BPh) onto the tissue sections and intact worms, $230 \mu \mathrm{L}$ of a $20 \mathrm{mg} / \mathrm{mL}$ solution in acetonitrile:2-propanol:water 6:3:1 + 0.5\% TFA were pneumatically sprayed using an automated sample preparation robot (SMALDIPrep, TransMIT GmbH, Giessen, Germany). Resulting matrix crystal sizes on tissue were on average below $10 \mu \mathrm{m}$ (Figure S2A). 2,5-dihydroxybenzoic acid (DHB) was applied by spraying $85 \mu \mathrm{L}$ of a $30 \mathrm{mg} / \mathrm{mL}$ solution in acetone:water $1: 1+0.2 \%$ TFA. Matrix solutions were sprayed for 23 min and 8 min $30 \mathrm{~s}$ (flow rate setting $10 \mu \mathrm{L} / \mathrm{min}$ ), respectively. For comparison of the MALDI matrices BPh and DHB, 
one half of a mouse brain-section was sprayed with DHB and the other with BPh. To promote alkali metal adduct formation and allow comparison to DHB experiments, 0.1 mmol sodium carbonate and potassium acetate were added to BPh. UV irradiation experiments during matrix application were performed using an UV pencil lamp $\left(254 \mathrm{~nm}, 4.5 \mathrm{~mW} / \mathrm{cm}^{2}\right.$; Edmund Optics, York, UK) placed inside the MALDI sample-preparation spray chamber as shown in Figure S2B. The lamp-sample distance was $3 \mathrm{~mm}$ and the sample was irradiated by UV light for 3 min. The irradiation was started with a time delay of $15 \mathrm{~s}$.

PL standards and the SM brain extract were deposited on MALDI targets either by pneumatic spray deposition of a $0.1-1.0 \mathrm{mM} \mathrm{PL}$ standard/SM brain extract mixed with $20 \mathrm{mg} / \mathrm{mL} \mathrm{BPh}$ in $230 \mu \mathrm{L}$ acetonitrile:2-propanol:water 6:3:1 + 0.5\% TFA or by dried-droplet deposition of the lipid standard solution before pneumatically spraying BPh solution (Supporting Protocol 1). To test the impact of matrix application methods for BPh on MALDI-MS results, we performed matrix sublimation in a sublimation chamber. The design of the application chamber was similar to the setup described by Murphy and co-workers ${ }^{4}$ and the setup was previously employed by us for other matrices. ${ }^{5}$ For sublimation of BPh, solid BPh deposited in the sublimation chamber was heated to $40^{\circ} \mathrm{C}$ at $0.5 \mathrm{mbar}$ for $10 \mathrm{~min}$. To assist crystallization of the vapor phase on the tissue section, the condenser was filled with ice water. After sublimation, the tissue section was carefully removed from the sublimation chamber, optical microscopy and MALDI-MS were performed as described below in "MALDI mass spectrometry imaging".

\section{MALDI mass spectrometry imaging}

After matrix application, MALDI-MS, MALDI-MSI, MALDI-MS ${ }^{2}$ and MALDI-MS ${ }^{2}$ I experiments were performed employing an AP-SMALDI5-AF mass spectrometry imaging ion source (TransMIT GmbH, Giessen, Germany) coupled to an orbital trapping MS (Q Exactive HF, Thermo Fisher Scientific GmbH, Bremen, Germany). For all MALDI-MS, MALDI-MSI, MALDI$M^{2}$ and MALDI-MS'I experiments the full-pixel mode was used. In full-pixel mode, the square region of a defined pixel is rasterized by quickly moving the sample spot in a meandering 
fashion, while the laser is firing with a high repetition rate. This scan mode results in almost complete ablation of the selected pixel area. The instrumental parameters of the mass spectrometer for all MS- and $\mathrm{MS}^{2}$-experiments were as follows: mass resolution at $\mathrm{m} / \mathrm{z} 200$ 240,000, normalized collision energy setting - between 16 and 18; inlet capillary temperature - $250{ }^{\circ} \mathrm{C}$; S-lens setting - 100. For DHB, matrix clusters were used for internal mass calibration, whereas for BPh external mass calibration was performed using the protonated PC 34:1 ion signal.

Laser energies were measured with a laser power meter (FieldMaxII-TOP, Coherent, Dieburg, Germany) after all optical elements. For S. mansoni measurements the recently developed autofocusing technology was used to avoid height related measurement artefacts. ${ }^{6}$ Supporting Protocol 2 was used for hematoxylin and eosin (H\&E) staining. For all microscope images, a Keyence VHX-5000 digital microscope (Keyence Deutschland GmbH, NeuIsenburg, Germany) was employed. UV/Vis measurements of matrix-covered microscopy slides (VWR microscopy slides, Leuven, Belgium) were performed with a Lambda20 (PerkinElmer, Buckinghamshire, UK) instrument.

\section{Data analysis and visualization}

MS data were analyzed with the Thermo XCalibur Qual Browser for direct spectrum analysis and with the MSiReader imaging software ${ }^{7}$ for MS image processing. All images were created with a $\mathrm{m} / \mathrm{z}$ tolerance of $\pm 10 \mathrm{ppm}$. Ion-signal intensities used for MALDI-MSI were normalized to the total ion current (TIC) per pixel and to the highest signal intensity per image. For the DBposition isomer resolved MALDI-MS²I, ion-signal intensities of the DB-associated aldehydefragment ions of the respective isomers were normalized to the TIC or ratios of ion intensities were used. Lipids in MALDI-MSI experiments were assigned by comparing accurate (experimental) mass values, to exact (theoretical) mass values using data bank searches in LIPID MAPS. Apparent reaction yields, $Y$, were calculated as (mass spectrometric intensity of 
PB product ion)/(mass spectrometric intensity of PB product ion + mass spectrometric intensity of precursor ion) $\cdot 100 \%$.

$Y$ for PB product ion does not strongly depend on the number of laser pulses, indicating that every laser pulse generates on average the same relative amount of endogenous lipid and PB product ions. Therefore, the PB reaction initiated by one laser pulse must be completed before the next laser pulse is triggered. This suggests that the minimum PB reaction time during MALDI is $1 \mathrm{~ns}$, the laser pulse length, and the maximum reaction time can be estimated to be $2.5 \mathrm{~ms}$ due to the use of $400 \mathrm{~Hz}$ laser repetition rate in all experiments.

\section{Lipid extraction, HPLC-ESI-MS ${ }^{2}$ and shotgun PB-ESI-MS ${ }^{2}$}

The methyl-tert-butylether (MTBE)-extraction of the mouse cerebellum-tissue section after BPh-application was done according to the protocol introduced by Matyash et al.. ${ }^{8}$ First, the tissue was scratched off the glass slide and transferred into a MTBE-rinsed Eppendorf cup. Subsequently, $20 \mu \mathrm{L}$ of a $0.1 \% \mathrm{NH}_{4} \mathrm{Ac}$-solution were added and the mixture was homogenized 3 times for 7 min at 30 rotations/s in a zirconia bead mill (Mixer Mill MM 301, Retsch GmbH, Haan, Germany). MeOH $(70 \mu \mathrm{L})$ and MTBE $(280 \mu \mathrm{L})$ were added and the mixture was vortexed at $4^{\circ} \mathrm{C}$ and 950 rpm for $1 \mathrm{~h}$ (ThermoMixer C, Eppendorf, Hamburg, Germany) before $140 \mu \mathrm{L}$ ice-cold water was added. The resulting solution was centrifuged for $10 \mathrm{~min}$ at $1000 \mathrm{G}$ (Centrifuge 5804, Eppendorf, Hamburg, Germany). Subsequently the upper phase (MTBE) was removed and stored, while the aqueous lower phase was reextracted by adding $140 \mu \mathrm{L}$ of MTBE:MeOH: $\mathrm{H}_{2} \mathrm{O} 4: 1.2: 1$. The mixture was vortexed for $1 \mathrm{~h}$ at $4{ }^{\circ} \mathrm{C}$ and $1000 \mathrm{rpm}$ followed by 10 min centrifugation at $1000 \mathrm{G}$. After the resulting two MTBE-phases were combined and placed in an ice bath, the solvent was evaporated under $\mathrm{N}_{2}$-stream. The dried extract $(5.210 \mathrm{mg})$ was resolved in $120 \mu \mathrm{L}$ acetonitrile, $100 \mu \mathrm{L} \mathrm{MeOH}, 80 \mu \mathrm{L}$ water and $50 \mu \mathrm{L}$ chloroform.

For HPLC analysis, a Dionex UltiMate 3000 (Thermo Fisher Scientific GmbH, Bremen, Germany) equipped with a $C_{18} 2.1 \mathrm{~mm} \times 100 \mathrm{~mm}(2.6 \mu \mathrm{m}$ particle size) column (Kinetex phenomenex) was used in combination with an ESI source coupled to a Q Exactive HF-X 
(Thermo Fisher Scientific GmbH, Bremen, Germany) mass spectrometer. A binary gradient system partly adapted from $\mathrm{Hu}$ et al. $^{9}$ of 60:40 acetonitrile:water and 90:8:2 2-propanol:acetonitrile:water, both containing $0.1 \%$ formic acid and $10 \mathrm{mmol} / \mathrm{L}$ ammonium formate, was used as eluent $A$ and $B$, respectively. The elution was performed with a gradient of $32 \mathrm{~min}$; during 0 to $1.5 \mathrm{~min}$ isocratic elution with $32 \% \mathrm{~B}$, from 1.5 to $4 \mathrm{~min}$ increase to $45 \%$ B, from 4 to 5 min to $52 \% \mathrm{~B}$, from 5 to $8 \mathrm{~min}$ to $58 \% \mathrm{~B}$, from 8 to $11 \mathrm{~min}$ to $66 \% \mathrm{~B}$, from 11 to $14 \mathrm{~min}$ to $70 \% \mathrm{~B}$, from 14 to $18 \mathrm{~min}$ to $75 \% \mathrm{~B}$, from 18 to $21 \mathrm{~min}$ to $97 \% \mathrm{~B}$, during 21 to $25 \mathrm{~min}$ $97 \%$ was maintained, from 25 to 26 min solvent B was decreased to $32 \%$ and maintained at $32 \%$ from 26 to $32 \mathrm{~min}$. The flow rate was $260 \mu \mathrm{L} / \mathrm{min}$ and the column oven temperature was maintained at $50^{\circ} \mathrm{C}$. A precolumn was used for all chromatographic runs. Extract volumes of $10 \mu \mathrm{L}$ were injected and analyzed in positive-ion mode $(\mathrm{m} / \mathrm{z} 600-1000)$ for $\mathrm{MS}^{1}$ and the $\mathrm{m} / \mathrm{z}$ range was automatically adjusted for $\mathrm{MS}^{2}$ experiments. Three replicate measurements were performed. Settings for all experiments were: mass resolution 240,000 at $\mathrm{m} / \mathrm{z} 200$, normalized collision energy setting of 18 , a sheath gas flow rate of 35 , an aux gas flow rate of 12 , a spray voltage of $3.5 \mathrm{kV}, 300^{\circ} \mathrm{C}$ inlet capillary temperature, a funnel RF level of 70 and an aux gas heater temperature of $150^{\circ} \mathrm{C}$. All ions listed in Table S3 were included in the inclusion list for $\mathrm{MS}^{2}$ experiments with dynamic exclusion of $10 \mathrm{~s}$. $Y$ values for PB products were calculated by replacing ion intensities by chromatographic peaks in the above given formula.

In order to assign the fatty acid composition and confirm DB-position assignments, lipids were extracted from about 100 mating-experienced male S. mansoni worms. Worms were homogenized in $25 \mu \mathrm{L} 0.1 \% \mathrm{NH}_{4} \mathrm{Ac}$ (ice bath cooling employed). After addition of $100 \mu \mathrm{L} \mathrm{MeOH}$ and $400 \mu \mathrm{L}$ MTBE, the mixture was vortexed for $1 \mathrm{~h}$ at $4^{\circ} \mathrm{C}$ at $500 \mathrm{rpm}$. Subsequently, $200 \mu \mathrm{L}$ ice cooled water was added and the mixture was centrifuged for $10 \mathrm{~min}$ at $1000 \mathrm{G}$. The organic phase was collected and the aqueous phase was reextracted with MTBE as described above. The organic phases were combined and solvent was removed by $\mathrm{N}_{2}$ stream. Lipids were resuspended in $\mathrm{CHCl}_{3}$ and stored at $-20^{\circ} \mathrm{C}$ until use. For negative- and positive-ion mode $100 \mu \mathrm{L}$ extract were dried and resuspended in $49 \mu \mathrm{L} \mathrm{H}_{2} \mathrm{O} / 50 \mu \mathrm{L}$ acetone $+1 \mu \mathrm{L} 0.5 \mathrm{M}$ aqueous ammonium formate solution and $49 \mu \mathrm{L} \mathrm{H}_{2} \mathrm{O} / 50 \mu \mathrm{L}$ acetone $+1 \mu \mathrm{L} 0.5 \mathrm{M}$ formic acid, 
respectively. Resulting solutions were analyzed in negative/positive ion mode (spray voltage $\pm 1.3 \mathrm{kV}$ ) by nanoESI-MS ${ }^{2}$ on a $\mathrm{Q}$ Exactive mass spectrometer (mass resolution 120,000 at $m / z$ 200). In order to perform DB-position assignment, PB reactions between PLs and acetone were initiated with a $254 \mathrm{~nm}$ mercury lamp integrated into the nanoESI ion source as described in ref. ${ }^{10}$. HCD was set to $14-25$ in all experiments.

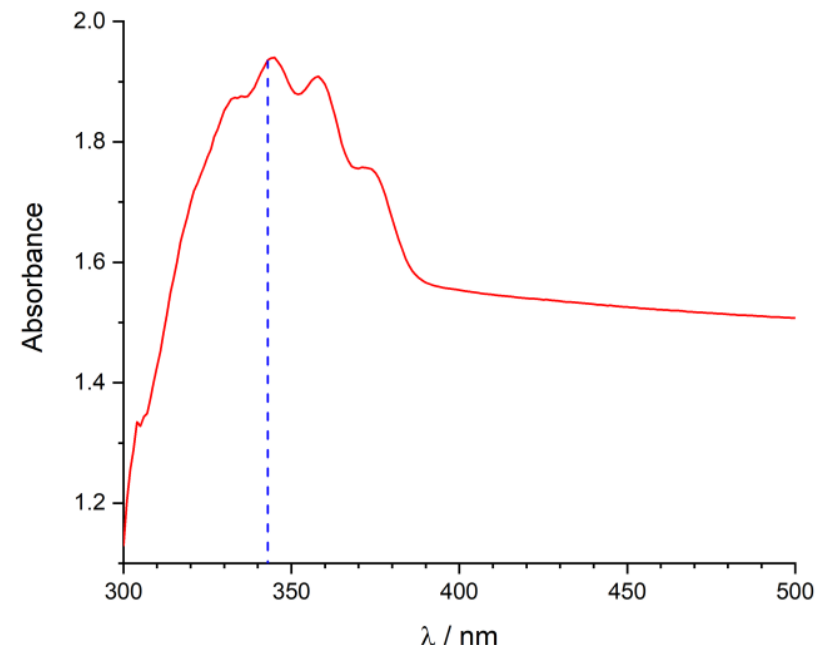

Figure S1. UV/Vis spectrum of BPh-covered microscopy slide between 300-500 nm. An untreated glass slide was used as reference. The absorbance at around 1.6 between $400-500 \mathrm{~nm}$ stems from diffuse reflection losses of the glass substrate. The blue line indicates the wavelength of the MALDI laser used in this study.

A

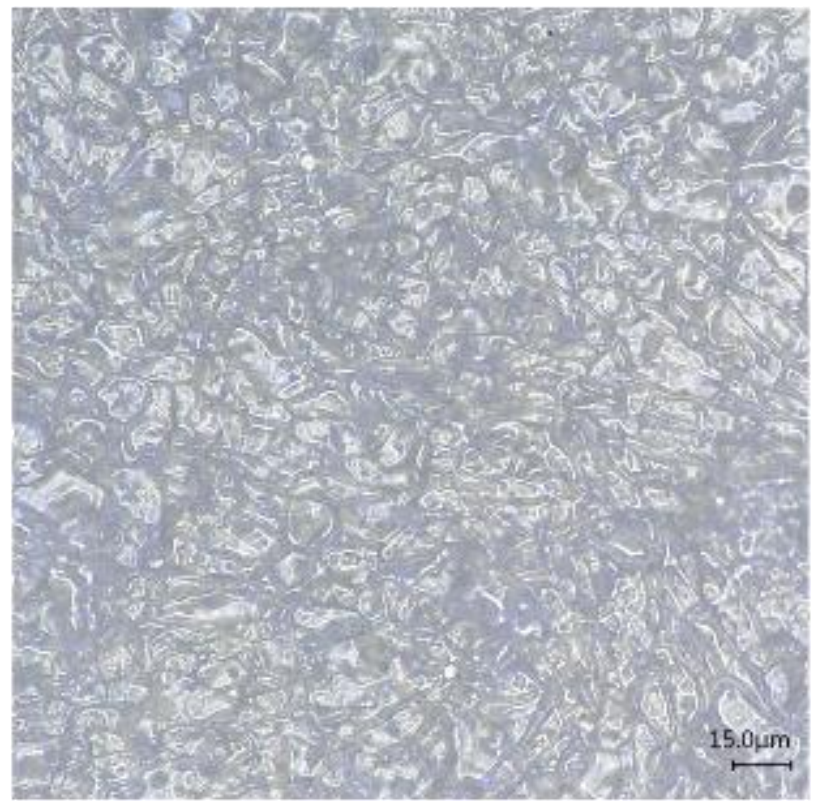

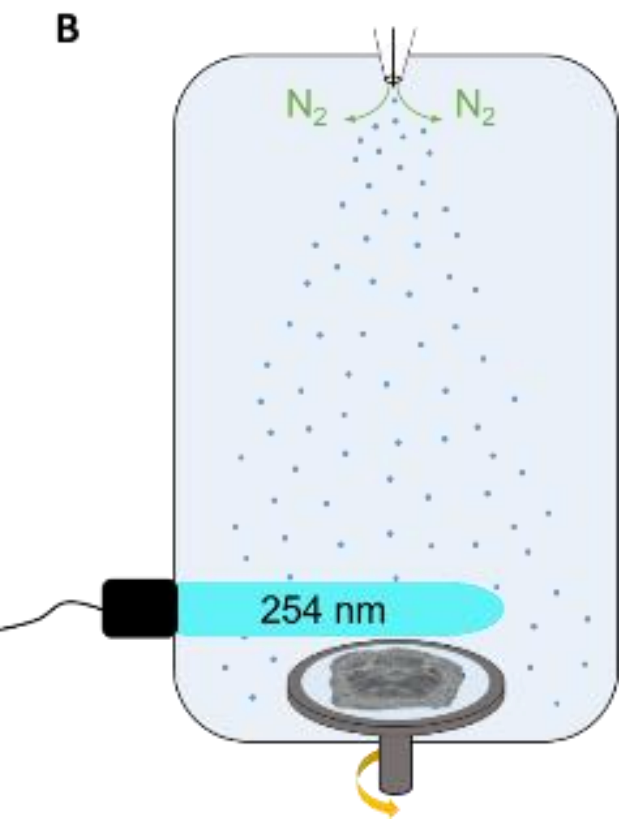

Figure S2. A: Microscopic image of BPh crystals on a mouse cerebellum-tissue section after pneumatic spray deposition according to Supporting Protocol 1. B: Pneumatic BPh spraying setup for UV irradiation of a sample during pneumatic matrix application. The $\mathrm{Hg}$-vapor lamp was positioned $3 \mathrm{~mm}$ above the rotating sample holder. 
Table S1. Assigned lipids in positive ion mode are listed with experimental $\mathrm{m} / \mathrm{z}$ values of detected adducts. PLs exclusively detected with BPh as matrix (blue), exclusively detected with DHB as matrix (red) and detected with both matrices (black) are grouped. Corresponding adducts and the mass error relative to the theoretical $\mathrm{m} / \mathrm{z}$ value of assigned compounds are listed. For table entries labelled with $< \pm 0.1 \mathbf{p p m}$, the absolute mass error was below $0.1 \mathrm{ppm}$.

\begin{tabular}{|c|c|c|c|c|c|c|c|c|}
\hline \multirow{3}{*}{$\begin{array}{l}\text { Lipid } \\
\text { PA 40:7 }\end{array}$} & \multicolumn{8}{|c|}{ Accurate $m / z$ (error / ppm) } \\
\hline & \multicolumn{2}{|c|}{$\mathbf{H}^{+}$} & \multicolumn{2}{|c|}{$\mathrm{Na}^{+}$} & \multicolumn{2}{|c|}{$\mathbf{K}^{+}$} & \multicolumn{2}{|c|}{$\mathrm{NH}_{4}{ }^{+}$} \\
\hline & & & 769.4779 & $(< \pm 0.1)$ & 785.4518 & $(< \pm 0.1)$ & & \\
\hline PA 42:7 & & & & & 813.4831 & $(< \pm 0.1)$ & & \\
\hline PA 42:8 & & & & & 811.4674 & $(-0.1)$ & & \\
\hline PC 30:1 & & & & & 742.4783 & $(-0.1)$ & & \\
\hline PC 36:0 & & & & & 828.5879 & $(< \pm 0.1)$ & & \\
\hline PC 40:1 & & & & & 882.6348 & $(-0.1)$ & & \\
\hline PC 40:2 & & & & & 880.6192 & $(< \pm 0.1)$ & & \\
\hline PC 40:4 & & & & & 876.5879 & $(< \pm 0.1)$ & & \\
\hline PC 42:1 & & & & & 910.6661 & $(-0.1)$ & & \\
\hline PC 42:2 & & & & & 908.6505 & $(< \pm 0.1)$ & & \\
\hline PC 42:7 & & & & & 898.5722 & $(-0.1)$ & & \\
\hline PC 44:12 & & & & & 916.5253 & $(< \pm 0.1)$ & & \\
\hline PE 40:0 & & & 826.6296 & $(< \pm 0.1)$ & & & & \\
\hline PE 42:6 & & & & & 858.5409 & $(-0.1)$ & & \\
\hline PG 34:1 & & & & & & & 766.5594 & $(+0.1)$ \\
\hline PG 38:0 & & & & & & & 824.6376 & $(+0.1)$ \\
\hline PG 40:0 & & & & & & & 852.6689 & $(+0.1)$ \\
\hline PG 40:1 & & & & & & & 850.6533 & $(+0.1)$ \\
\hline PS 38:0 & 820.6063 & $(+0.1)$ & & & & & & \\
\hline PS 40:6 & & & & & 874.4995 & $(< \pm 0.1)$ & & \\
\hline PS 42:5 & & & & & 904.5464 & $(< \pm 0.1)$ & & \\
\hline $\mathrm{SM} d 42: 3$ & & & & & 849.6246 & $(< \pm 0.1)$ & & \\
\hline TG 50:1 & & & & & 871.7151 & $(< \pm 0.1)$ & & \\
\hline TG 52:2 & & & 881.7568 & $(-0.1)$ & 897.7308 & $(< \pm 0.1)$ & & \\
\hline TG 52:3 & & & & & 895.7151 & $(< \pm 0.1)$ & & \\
\hline PC 34:3 & 756.5538 & $(< \pm 0.1)$ & & & & & & \\
\hline PC 40:8 & 830.5694 & $(< \pm 0.1)$ & & & & & & \\
\hline PE 38:2 & 772.5851 & $(< \pm 0.1)$ & & & & & & \\
\hline PS $39: 3$ & 828.5749 & $(< \pm 0.1)$ & & & & & & \\
\hline PS 0-36:1 / PS P-36:0 & 776.5800 & $(< \pm 0.1)$ & & & & & & \\
\hline PS 0-36:2 / PS P-36:1 & 774.5643 & $(< \pm 0.1)$ & & & & & & \\
\hline PS 0-38:2 / PS P-38:1 & 802.5956 & $(< \pm 0.1)$ & & & & & & \\
\hline PA $34: 1$ & & & & & 713.4518 & $(< \pm 0.1)$ & & \\
\hline PA $34: 2$ & & & & & 711.4361 & $(-0.1)$ & & \\
\hline PA 35:1 & & & 711.4935 & $(< \pm 0.1)$ & & & & \\
\hline PA $36: 1$ & & & 725.5092 & $(< \pm 0.1)$ & 741.4831 & $(< \pm 0.1)$ & & \\
\hline PA 36:2 & & & 723.4935 & $(< \pm 0.1)$ & 739.4674 & $(-0.1)$ & & \\
\hline PA $36: 3$ & & & & & 737.4518 & $(< \pm 0.1)$ & & \\
\hline PA 38:2 & & & 751.5248 & $(< \pm 0.1)$ & 767.4987 & $(-0.1)$ & & \\
\hline PA $38: 3$ & & & & & 765.4831 & $(< \pm 0.1)$ & & \\
\hline PA 38:5 & & & & & 761.4518 & $(< \pm 0.1)$ & & \\
\hline PA 40:4 & & & 775.5248 & $(< \pm 0.1)$ & & & & \\
\hline
\end{tabular}




\begin{tabular}{|c|c|c|c|c|c|c|c|c|}
\hline \multicolumn{5}{|l|}{ PA 40:5 } & \multicolumn{4}{|c|}{$789.4831 \quad(< \pm 0.1)$} \\
\hline PA 40:6 & & & & & 787.4674 & $(-0.1)$ & & \\
\hline PC 32:0 & 734.5694 & $(< \pm 0.1)$ & 756.5514 & $(< \pm 0.1)$ & 772.5253 & $(< \pm 0.1)$ & & \\
\hline PC 32:1 & 732.5538 & $(< \pm 0.1)$ & & & 770.5096 & $(-0.1)$ & & \\
\hline PC 34:0 & 762.6007 & $(< \pm 0.1)$ & 784.5827 & $(< \pm 0.1)$ & 800.5566 & $(< \pm 0.1)$ & & \\
\hline PC 34:1 & 760.5851 & $(< \pm 0.1)$ & 782.5670 & $(< \pm 0.1)$ & 798.5409 & $(-0.1)$ & & \\
\hline PC 34:2 & 758.5694 & $(< \pm 0.1)$ & 780.5514 & $(< \pm 0.1)$ & 796.5253 & $(< \pm 0.1)$ & & \\
\hline PC 36:1 & 788.6164 & $(< \pm 0.1)$ & 810.5983 & $(< \pm 0.1)$ & 826.5722 & $(-0.1)$ & & \\
\hline PC 36:2 & 786.6007 & $(< \pm 0.1)$ & 808.5827 & $(< \pm 0.1)$ & 824.5566 & $(< \pm 0.1)$ & & \\
\hline PC $36: 3$ & 784.5851 & $(< \pm 0.1)$ & 806.5670 & $(< \pm 0.1)$ & 822.5409 & $(-0.1)$ & & \\
\hline PC $36: 4$ & 782.5694 & $(< \pm 0.1)$ & 804.5514 & $(< \pm 0.1)$ & 820.5253 & $(< \pm 0.1)$ & & \\
\hline PC 38:1 & 816.6477 & $(< \pm 0.1)$ & 838.6296 & $(< \pm 0.1)$ & 854.6035 & $(-0.1)$ & & \\
\hline PC 38:2 & 814.6320 & $(< \pm 0.1)$ & 836.615 & $(+1.0)$ & 852.5879 & $(< \pm 0.1)$ & & \\
\hline PC 38:3 & & & 834.5983 & $(< \pm 0.1)$ & 850.5722 & $(-0.1)$ & & \\
\hline PC 38:4 & 810.6007 & $(< \pm 0.1)$ & 832.5827 & $(< \pm 0.1)$ & 848.5566 & $(< \pm 0.1)$ & & \\
\hline PC 38:5 & 808.5851 & $(< \pm 0.1)$ & 830.5672 & $(+0.2)$ & 846.5409 & $(-0.1)$ & & \\
\hline PC 38:6 & 806.5694 & $(< \pm 0.1)$ & 828.5514 & $(< \pm 0.1)$ & 844.5253 & $(< \pm 0.1)$ & & \\
\hline PC 38:7 & 804.5538 & $(< \pm 0.1)$ & & & 842.5096 & $(-0.1)$ & & \\
\hline PC 40:6 & 834.6007 & $(< \pm 0.1)$ & 856.5827 & $(< \pm 0.1)$ & 872.5566 & $(< \pm 0.1)$ & & \\
\hline PC 40:7 & 832.5851 & $(< \pm 0.1)$ & 854.5670 & $(< \pm 0.1)$ & 870.5409 & $(-0.1)$ & & \\
\hline PC 40:9 & 828.5538 & $(< \pm 0.1)$ & & & & & & \\
\hline PE 33:0 & & & & & 744.4940 & $(< \pm 0.1)$ & & \\
\hline PE 34:0 & & & 742.5357 & $(< \pm 0.1)$ & 758.5096 & $(-0.1)$ & & \\
\hline PE 34:1 & 718.5381 & $(< \pm 0.1)$ & & & 756.4940 & $(< \pm 0.1)$ & & \\
\hline PE 36:0 & 748.5851 & $(< \pm 0.1)$ & 770.5670 & $(< \pm 0.1)$ & 786.5409 & $(-0.1)$ & & \\
\hline PE $36: 1$ & 746.5694 & $(< \pm 0.1)$ & & & 784.5253 & $(< \pm 0.1)$ & & \\
\hline PE 36:2 & 744.5538 & $(< \pm 0.1)$ & & & 782.5096 & $(-0.1)$ & & \\
\hline PE 38:1 & 774.6007 & $(< \pm 0.1)$ & & & 812.5566 & $(< \pm 0.1)$ & & \\
\hline PE 38:4 & & & & & 806.5096 & $(-0.1)$ & & \\
\hline PE 38:5 & & & & & 804.4940 & $(< \pm 0.1)$ & & \\
\hline PE 38:6 & & & 786.5046 & $(+0.2)$ & 802.4783 & $(-0.1)$ & & \\
\hline PE 38:6(OH) & & & & & 818.4733 & $(< \pm 0.1)$ & & \\
\hline PE 40:4 & & & & & 834.5409 & $(-0.1)$ & & \\
\hline PE 40:6 & 792.5538 & $(< \pm 0.1)$ & 814.5357 & $(< \pm 0.1)$ & 830.5096 & $(-0.1)$ & & \\
\hline PE 40:7 & 790.5381 & $(< \pm 0.1)$ & 812.5201 & $(< \pm 0.1)$ & 828.4940 & $(< \pm 0.1)$ & & \\
\hline PE 42:9 & 814.5381 & $(< \pm 0.1)$ & & & & & & \\
\hline PE P-36:2 & & & & & 766.5147 & $(< \pm 0.1)$ & & \\
\hline PG 34:0 & & & 773.5303 & $(< \pm 0.1)$ & & & & \\
\hline PG 36:0 & & & 801.5616 & $(< \pm 0.1)$ & & & & \\
\hline PG 36:1 & & & 799.5459 & $(-0.1)$ & & & & \\
\hline PG 38:1 & & & 827.5772 & $(-0.1)$ & & & 822.6220 & $(+0.1)$ \\
\hline PG 38:4 & & & 821.5303 & $(< \pm 0.1)$ & & & & \\
\hline PI 38:4 & & & & & 925.5203 & $(< \pm 0.1)$ & & \\
\hline PS $36: 0$ & 792.5749 & $(< \pm 0.1)$ & & & 830.5308 & $(< \pm 0.1)$ & & \\
\hline PS $36: 1$ & 790.5592 & $(-0.1)$ & & & 828.5151 & $(< \pm 0.1)$ & & \\
\hline PS 36:2 & & & & & 826.4995 & $(< \pm 0.1)$ & & \\
\hline PS 40:0 & 848.6375 & $(< \pm 0.1)$ & & & & & & \\
\hline
\end{tabular}




\begin{tabular}{lllllll} 
SM d34:0 & & & & 743.5464 & $(< \pm 0.1)$ \\
SM d34:1 & 703.5748 & $(< \pm 0.1)$ & 725.5568 & $(< \pm 0.1)$ & 741.5307 & $(< \pm 0.1)$ \\
SM d36:1 & 731.6061 & $(< \pm 0.1)$ & 753.5881 & $(< \pm 0.1)$ & 769.5620 & $(< \pm 0.1)$ \\
SM d38:1 & & & 781.6194 & $(< \pm 0.1)$ & 797.5933 & $(< \pm 0.1)$ \\
SM d38:2 & 757.6218 & $(< \pm 0.1)$ & 779.6037 & $(< \pm 0.1)$ & 795.5777 & $(< \pm 0.1)$ \\
SM d40:1 & 787.6687 & $(< \pm 0.1)$ & 809.6507 & $(< \pm 0.1)$ & 825.6246 & $(< \pm 0.1)$ \\
SM d40:2 & 785.6531 & $(< \pm 0.1)$ & 807.6350 & $(< \pm 0.1)$ & 823.6090 & $(< \pm 0.1)$ \\
SM d41:2 & & & 821.6507 & $(< \pm 0.1)$ & & \\
SM d42:1 & & & 837.6820 & $(< \pm 0.1)$ & 853.6559 & $(< \pm 0.1)$ \\
SM d42:2 & 813.6844 & $(< \pm 0.1)$ & 835.6663 & $(< \pm 0.1)$ & 851.6403 & $(< \pm 0.1)$ \\
\hline
\end{tabular}

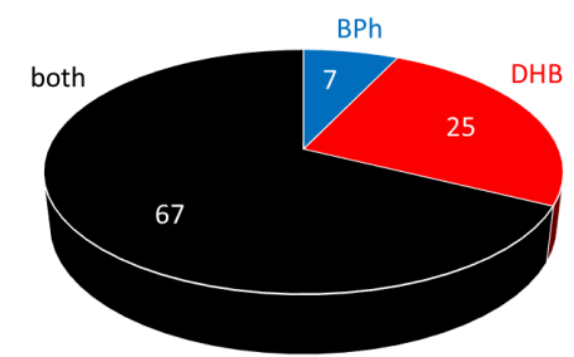

Figure S3. Total number of detected lipids in MALDI-MS experiments of mouse cerebellum. The black fraction of the diagram indicates lipids detected with both MALDI matrices, whereas the blue and red portion represent the number of lipids only detected with BPh or DHB, respectively.

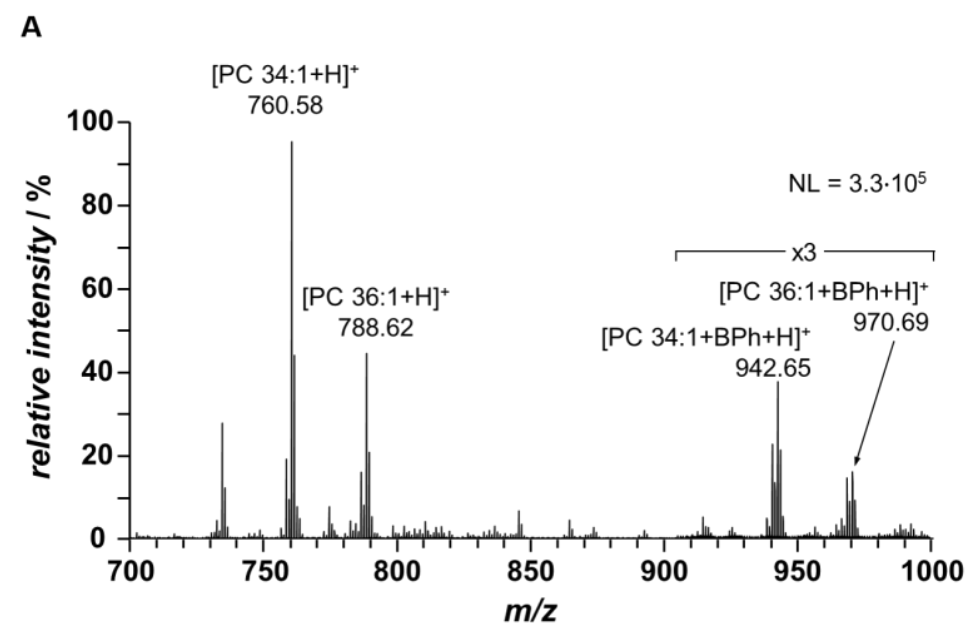

B

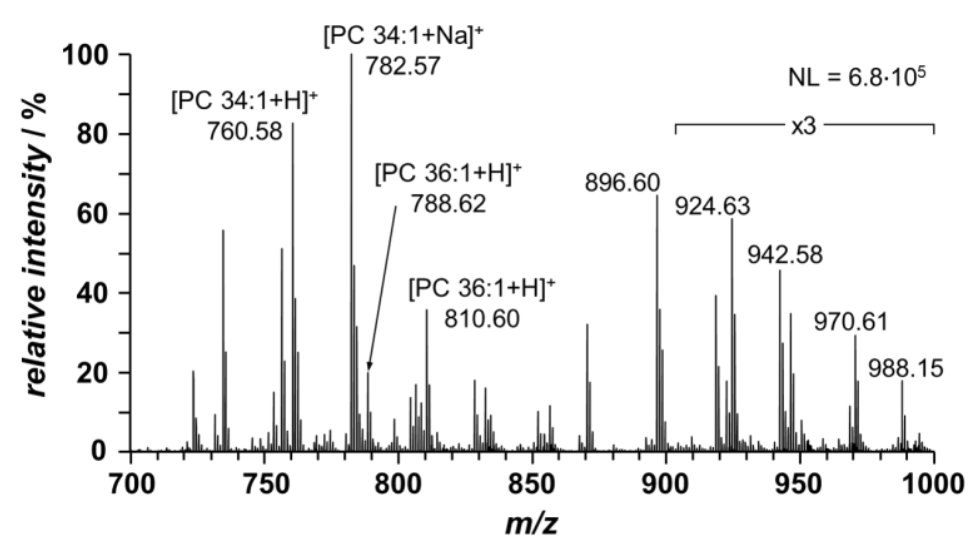

Figure S 4. Comparison of MALDI mass spectra of mouse brain tissue sections covered by pneumatical spraying with (A) BPh and (B) DHB. No ion signals with the same $\mathrm{m} / \mathrm{z}$ values as in (A) are observed in (B). 


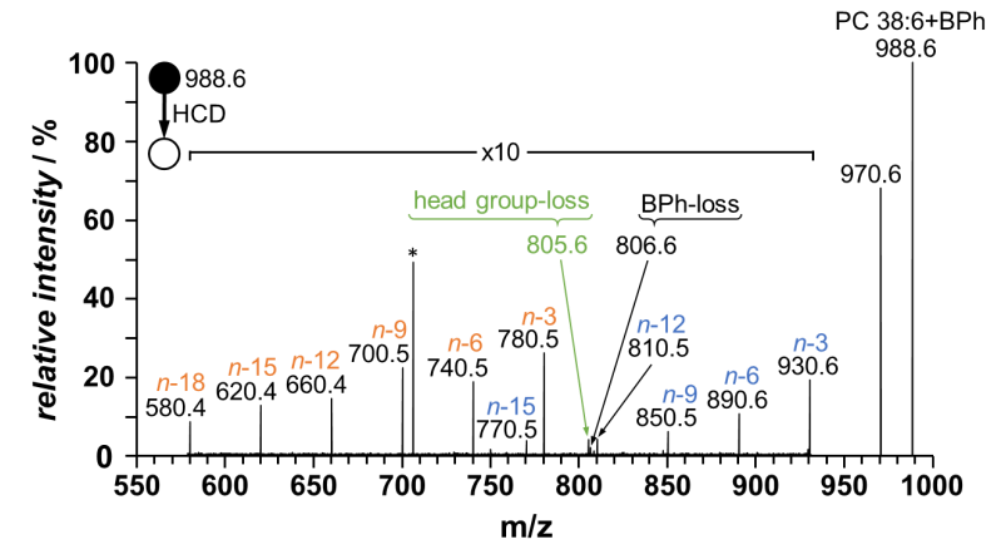

Figure S5. MALDI-HCD-MS ${ }^{2}$-spectrum of protonated PB product ions of PC 38:6 (n-18,n-15,n-12,n-9,n-6,n-3) acquired directly from a mouse brain section. DB fragment ion signals are labeled with the respective DB-positions. Aldehyde fragment ion signals are labeled in orange and diphenylethylene fragment ion signals in blue. The signal labeled with a star is electronic noise.

Table S2. PLs and corresponding PB product ions upon MALDI-MS of a BPh-covered mouse brain-section using pneumatical spraying (without UV lamp irradiation during the BPh application).

\begin{tabular}{|c|c|c|c|c|c|c|}
\hline \multicolumn{7}{|c|}{ Accurate $m / z$ (error / ppm) } \\
\hline \multirow{2}{*}{$\begin{array}{l}\text { Lipid } \\
\text { PA } 36: 2\end{array}$} & \multirow[t]{2}{*}{$\mathrm{H}^{+}$} & & \multicolumn{2}{|c|}{$\mathrm{Na}^{+}$} & \multicolumn{2}{|c|}{$\mathbf{K}^{+}$} \\
\hline & & & 723.4936 & $(< \pm 0.1)$ & 739.4676 & $(-0.1)$ \\
\hline PA 36:2+BPh & & & 905.5658 & $(-5.3)$ & 921.5397 & $(-5.8)$ \\
\hline PA 38:2 & & & & & 767.4986 & $(-0.1)$ \\
\hline PA 38:2+BPh & & & & & 949.5719 & $(+0.7)$ \\
\hline PC 32:1 & 732.5540 & $(< \pm 0.1)$ & & & 770.5096 & $(-0.1)$ \\
\hline $\mathrm{PC} 32: 1+\mathrm{BPh}$ & 914.6259 & $(-6.9)$ & & & 952.5827 & $(-0.4)$ \\
\hline PC 34:1 & 760.5850 & $(< \pm 0.1)$ & 782.5671 & $(< \pm 0.1)$ & 798.5409 & $(-0.1)$ \\
\hline PC 34:1+BPh & 942.6585 & $(+1.8)$ & 964.6406 & $(+1.8)$ & 980.6141 & $(+0.2)$ \\
\hline PC $34: 2$ & 758.5694 & $(< \pm 0.1)$ & 780.5517 & $(< \pm 0.1)$ & 796.5253 & $(< \pm 0.1)$ \\
\hline $\mathrm{PC} 34: 2+\mathrm{BPh}$ & 940.6425 & $(-0.4)$ & 962.6249 & $(+0.2)$ & 978.5984 & $(-0.4)$ \\
\hline PC 34:2 +2 BPh & 1122.7161 & $(+2.4)$ & & & 1160.6719 & $(+1.8)$ \\
\hline PC 34:3 & 756.5515 & $(-3.0)$ & & & & \\
\hline $\mathrm{PC} 34: 3+\mathrm{BPh}$ & 938.6257 & $(+5.7)$ & & & & \\
\hline PC $36: 1$ & 788.6163 & $(< \pm 0.1)$ & 810.5984 & $(< \pm 0.1)$ & 826.5722 & $(-0.1)$ \\
\hline $\mathrm{PC} 36: 1+\mathrm{BPh}$ & 970.6899 & $(+2.4)$ & 992.6728 & $(+6.8)$ & 1008.6457 & $(+1.8)$ \\
\hline PC $36: 2$ & 786.6007 & $(< \pm 0.1)$ & 808.5833 & $(< \pm 0.1)$ & 824.5566 & $(< \pm 0.1)$ \\
\hline $\mathrm{PC} 36: 2+\mathrm{BPh}$ & 968.6740 & $(+0.7)$ & 990.6569 & $(+2.4)$ & 1006.6297 & $(-0.4)$ \\
\hline PC 36:3 & & & & & 822.5409 & $(-0.1)$ \\
\hline PC 36:3+BPh & & & & & 1004.6142 & $(+0.7)$ \\
\hline PC $36: 4$ & & & & & 820.5253 & $(< \pm 0.1)$ \\
\hline PC $36: 4+B P h$ & & & & & 1002.5983 & $(-0.9)$ \\
\hline PC 38:1 & & & & & 854.6027 & $(-0.1)$ \\
\hline PC $38: 1+B P h$ & & & & & 1036.6767 & $(+4.6)$ \\
\hline PC 38:2 & 814.6320 & $(< \pm 0.1)$ & 836.6148 & $(+1.0)$ & 852.5868 & \\
\hline PC $38: 2+B P h$ & 996.7050 & $(-0.9)$ & 1018.6896 & $(+9.0)$ & 1034.6609 & $(+5.1)$ \\
\hline PC 38:4 & & & & & 848.5556 & $(< \pm 0.1)$ \\
\hline PC $38: 4+B P h$ & & & & & 1030.6297 & $(+5.1)$ \\
\hline PC $38: 5$ & & & & & 846.5401 & $(-0.1)$ \\
\hline PC 38:5+BPh & & & & & 1028.6143 & $(+5.7)$ \\
\hline
\end{tabular}




\begin{tabular}{lcccccc} 
PC 38:6 & 806.5690 & $(< \pm 0.1)$ & 828.5514 & $(< \pm 0.1)$ & 844.5243 & $(< \pm 0.1)$ \\
PC 38:6+BPh & 988.6424 & $(+1.3)$ & 1010.6250 & $(+2.6)$ & 1026.5985 & $(+5.7)$ \\
PC 38:7 & 804.5530 & $(< \pm 0.1)$ & & & 842.5086 & $(-0.1)$ \\
PC 38:7+BPh & 986.6262 & $(+0.2)$ & & & 1024.5826 & $(+4.6)$ \\
PC 40:6 & 834.6006 & $(< \pm 0.1)$ & 856.5820 & $(< \pm 0.1)$ & 872.5556 & $(< \pm 0.1)$ \\
PC 40:6+BPh & 1016.6743 & $(+2.9)$ & 1038.6560 & $(+4.6)$ & 1054.6299 & $(+6.2)$ \\
PC 40:7 & 832.5846 & $(< \pm 0.1)$ & 854.5663 & $(< \pm 0.1)$ & 870.5399 & $(-0.1)$ \\
PC 40:7+BPh & 1014.6578 & $(+0.2)$ & 1036.6402 & $(+4.0)$ & 1052.6141 & $(+5.7)$ \\
\hline
\end{tabular}

Table S3. Protonated PB-functionalized PLs from mouse cerebellum-tissue sections. Experimental $m / z$ values, confidently identified DB-positions with corresponding fragment ions are listed.

\begin{tabular}{|c|c|c|c|}
\hline Lipid & $\begin{array}{l}\text { Accurate } \mathbf{m} / \mathbf{z} \\
\text { of PB product }\end{array}$ & identified DB-positions & $\begin{array}{l}\text { aldehyde/diphenylethylene fragment ion } \\
\text { accurate } \mathrm{m} / \mathrm{z} \text { values (mass error / } \mathrm{ppm} \text { ) }\end{array}$ \\
\hline PC 32:1+BPh & 914.6285 & $(\mathrm{n}-7)$ & $(650.4407(+1.5) / 800.5243(+1.6))$ \\
\hline PC 34:2 (monounsat. FA)+BPh & 940.6411 & $(n-7) ;(n-9)$ & $\begin{array}{l}(676.4568(+2.1) / 826.5399(+1.5)) \\
(648.4253(+1.9) / 798.5086(+1.5))\end{array}$ \\
\hline PC $34: 2$ (diunsat. FA)+BPh & 940.6411 & $(n-6, n-9)$ & $\begin{array}{l}(690.4725(+2.2) / \mathrm{no}) \\
(650.4407(+1.5) / 800.5245(+1.8))\end{array}$ \\
\hline PC $34: 1+B P h$ & 942.6573 & $(n-7) ;(n-9)$ & $\begin{array}{l}(678.4723(+1.9) / 828.5552(+1.0)) \\
(650.4407(+1.5) / 800.5241(+1.3))\end{array}$ \\
\hline PC $36: 4$ (tetraunsat. FA)+BPh & 964.6389 & $(n-6, n-9, n-12, n-15)$ & $\begin{array}{l}(714.4723(+1.8) / 864.5547(+0.4), \\
674.4409(+1.8) / 824.5233(+0.3), \\
634.4099(+2.4) / 784.4926(+1.1), \\
594.3780(+1.5) / \mathrm{no})\end{array}$ \\
\hline PC 36:2 (monounsat. FA)+BPh & 968.6722 & $(n-6) ;(n-7) ;(n-9)$ & $\begin{array}{l}(718.5036(+1.8) / \mathrm{no}) \\
(704.4878(+1.6) / 854.5712(+1.4)) \\
(676.4566(+1.9) / 826.5395(+1.0))\end{array}$ \\
\hline PC $36: 1+B P h$ & 970.6874 & $(n-7) ;(n-9)$ & $\begin{array}{l}(706.5030(+1.0) / 856.5861(+0.5)) \\
(678.4720(+1.5) / 828.5549(+0.7))\end{array}$ \\
\hline $\mathrm{PC} 36: 2(\mathrm{OH})+\mathrm{BPh}$ & 984.6674 & $(n-7) ;(n-9)$ & $\begin{array}{l}(720.4830(+2.0) / \mathrm{no}) \\
(692.4516(+1.9) / 842.5347(+1.3))\end{array}$ \\
\hline $\mathrm{PC} 36: 1(\mathrm{OH})+\mathrm{BPh}$ & 986.6823 & $(n-9)$ & $(694.4669(+1.4) / \mathrm{no})$ \\
\hline PC 38:6+BPh & 988.6398 & $(n-3, n-6, n-9, n-12, n-15, n-18)$ & $\begin{array}{l}\text { (780.5191(+1.5)/930.6019(+0.7), } \\
740.4876(+1.3) / 890.5704(+0.5), \\
700.4564(+1.5) / 850.5395(+1.0) \\
660.4250(+1.4) / 810.5082(+1.0) \\
620.3939(+1.9) / 770.4776(+2.0), \\
580.3622(+1.3) / \mathrm{no})\end{array}$ \\
\hline
\end{tabular}

Table S4. Relative intensities of protonated endogenous PCs and the corresponding functionalized species, normalized to the ion intensities of protonated PC 34:1 and PC 34:1+BPh, respectively.

\begin{tabular}{|c|c|c|c|c|c|}
\hline Species & Accurate $\mathrm{m} / \mathbf{z}$ & $\frac{I}{I_{\mathrm{PC} 34: 1}} / \%$ & Species & Accurate $\mathrm{m} / \mathbf{z}$ & $\frac{I}{I_{\mathrm{PC} 34: 1+\mathrm{BPh}}} / \%$ \\
\hline PC 32:1 & 732.5540 & 4.7 & $\mathrm{PC} 32: 1+\mathrm{BPh}$ & 914.6259 & 13.9 \\
\hline PC 34:1 & 760.5850 & 100.0 & $\mathrm{PC} 34: 1+\mathrm{BPh}$ & 942.6585 & 100.0 \\
\hline PC 34:2 & 758.5694 & 20.0 & $\mathrm{PC} 34: 2+\mathrm{BPh}$ & 940.6425 & 59.5 \\
\hline PC $34: 3$ & 756.5515 & 2.7 & PC 34:3+BPh & 938.6257 & 12.8 \\
\hline PC 36:1 & 788.6163 & 47.0 & $\mathrm{PC} 36: 1+\mathrm{BPh}$ & 970.6899 & 41.8 \\
\hline PC 36:2 & 786.6007 & 17.2 & $\mathrm{PC} 36: 2+\mathrm{BPh}$ & 968.6740 & 38.9 \\
\hline
\end{tabular}




\begin{tabular}{lll|lll} 
PC 38:2 & 814.6320 & 2.9 & PC 38:2+BPh & 996.7050 & 4.5 \\
PC 38:6 & 806.5690 & 2.5 & PC 38:6+BPh & 988.6424 & 8.8 \\
PC 38:7 & 804.5530 & 1.9 & PC 38:7+BPh & 986.6262 & 6.0 \\
\hline
\end{tabular}

A

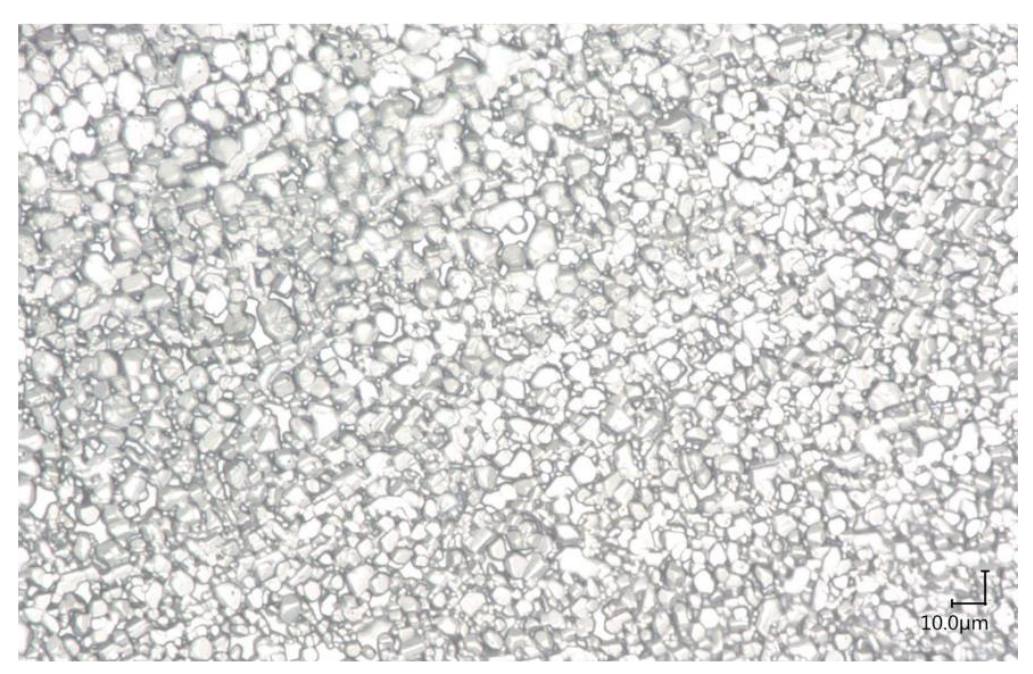

B

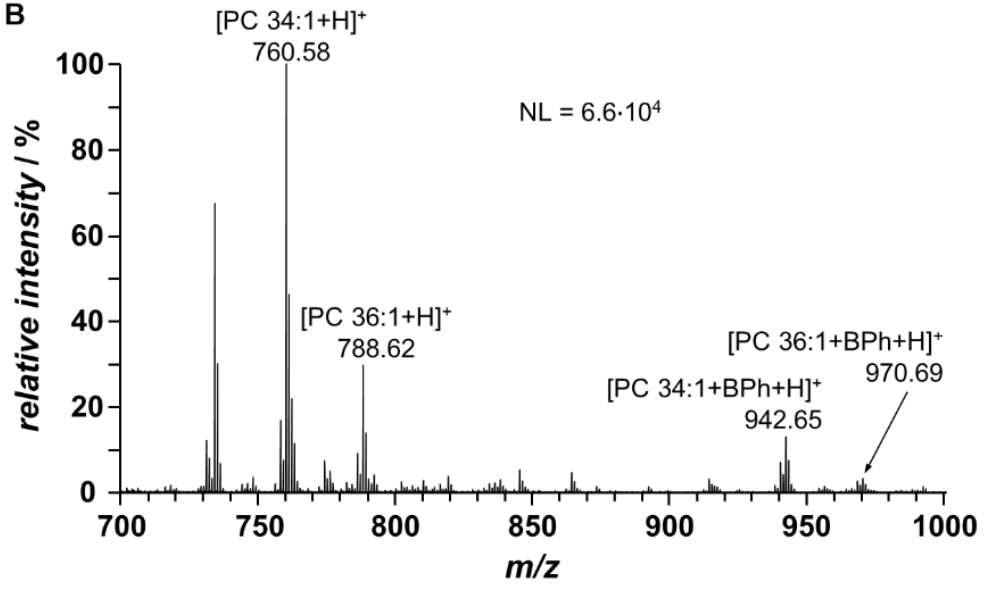

C

$[\mathrm{PC} 34: 1+\mathrm{BPh}+\mathrm{H}]^{+}$

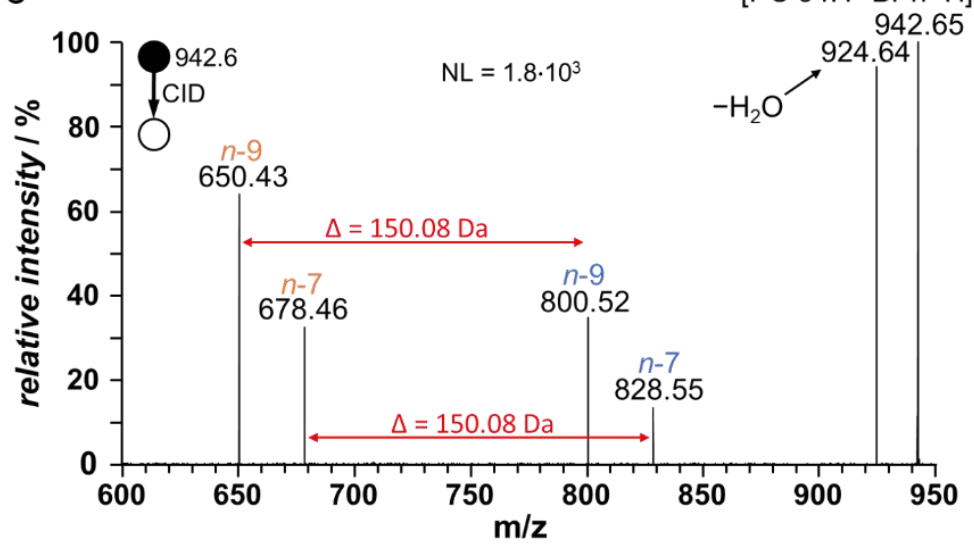

Figure S6. A: Microscopic image of BPh crystals on a mouse cerebellum-tissue section after sublimation of BPh. B. Positive ion mode MALDI mass spectrum for $\mathrm{m} / \mathrm{z} 700$ - 1000. Endogenous phospholipids and corresponding PB products were detected as protonated ions. Some signal assignments are included. C. MALDI-MS ${ }^{2}$ of $[\mathrm{PC} 34: 1+\mathrm{BPh}+\mathrm{H}]^{+}$from mouse brain tissue sections after sublimation of $\mathrm{BPh}$ confirming the presence of PB product ions. 


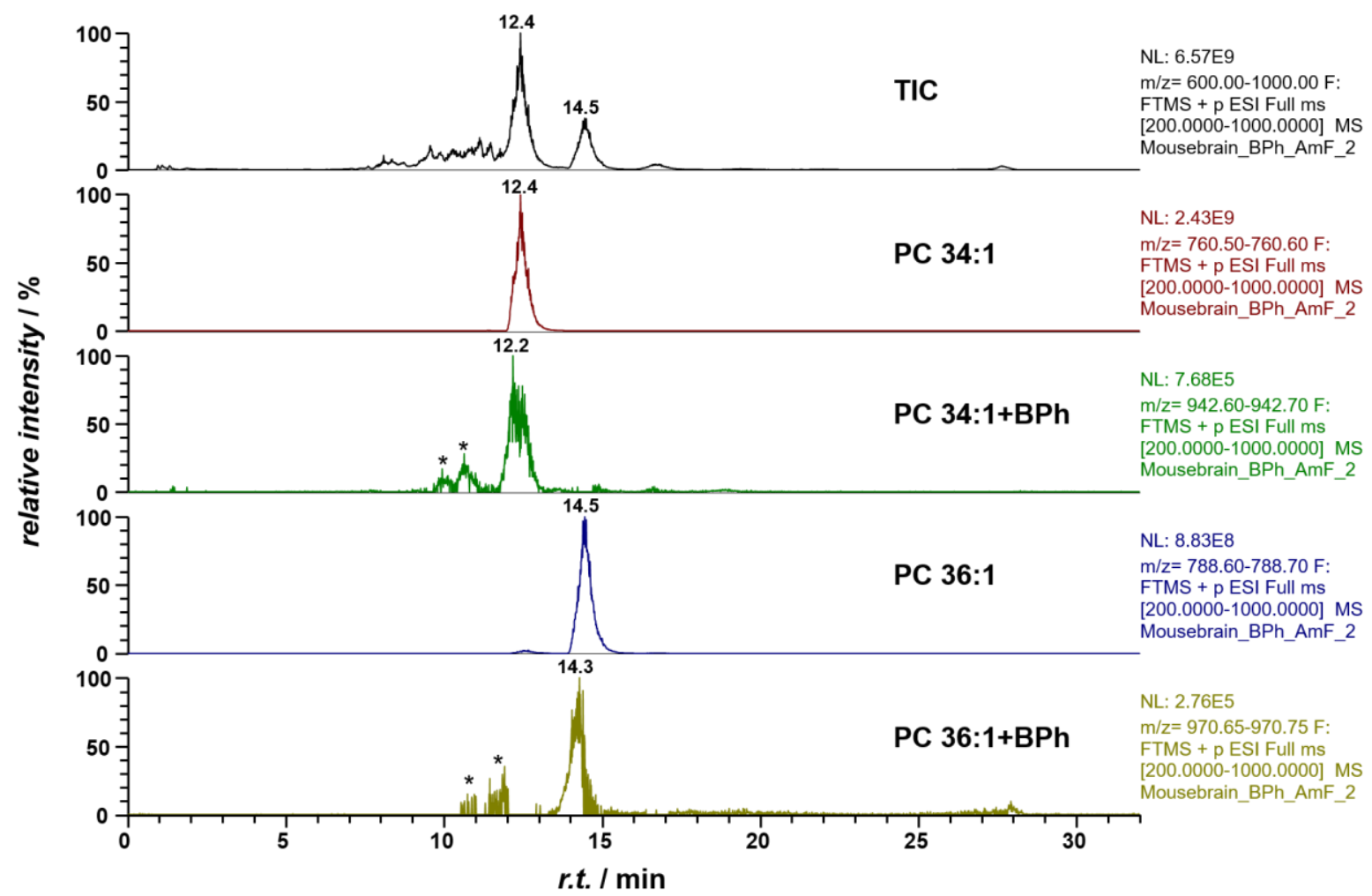

Figure S7. HPLC-ESI-MS and HPLC-ESI-MS ${ }^{2}$ of the MTBE-extract of mouse cerebellum tissue sections after pneumatic spraying of BPh solution. The $\mathrm{PB}$ reaction yield is about $0.03 \%$ for protonated $\mathrm{PC} 34: 1+\mathrm{BPh}$ and $\mathrm{PC} 36: 1+\mathrm{BPh}$. All compounds were detected as protonated ions. Similar reaction yields were obtained for all other PB products listed in Table S3. PB products were all confirmed by MS $^{2}$ experiments. ${ }^{*}-$ isobaric signals; no PB products based on ESI-MS² results.

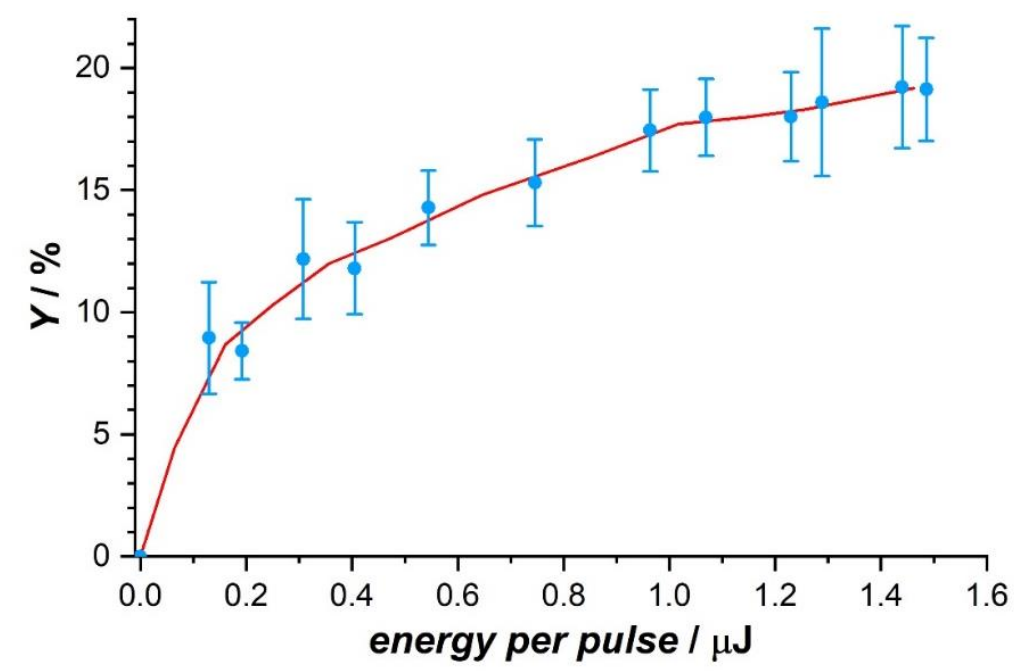

Figure S8. PB reaction yield, $Y$, of $[\mathrm{PC} 34: 1+\mathrm{BPh}+\mathrm{H}]^{+}$from $\mathrm{BPh}$ covered mouse brain-tissue as a function of the energy per MALDI laser pulse. The full pixel mode of the AP-SMALDI5-AF instrument with $25 \mu \mathrm{m}$ pixel size was used to keep the ablated area constant. Because the relative measure $Y$ is plotted as a function of laser energy per pulse, increased material ablation does not affect $Y$ as long as [PC $34: 1+\mathrm{H}]^{+}$and $[\mathrm{PC} 34: 1+\mathrm{BPh}+\mathrm{H}]^{+}$ have the same ionization efficiency. Blue dots represent the mean value of $Y$ (from 100 individual measurements). The error bars represent the corresponding standard deviation. The two-point floating average of the data points of $Y$ (red line) is shown as a guide to the eye. 


\section{A}

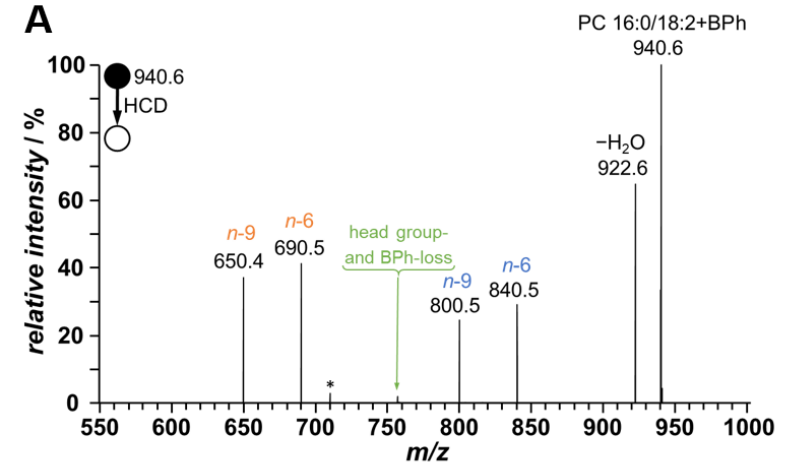

C

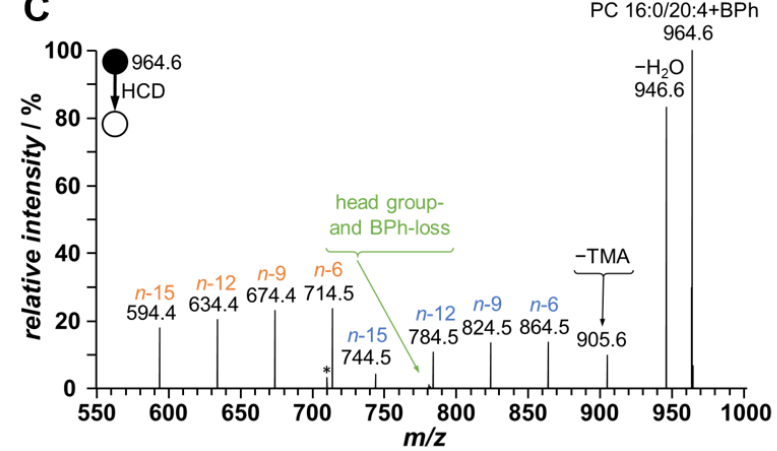

\section{E}

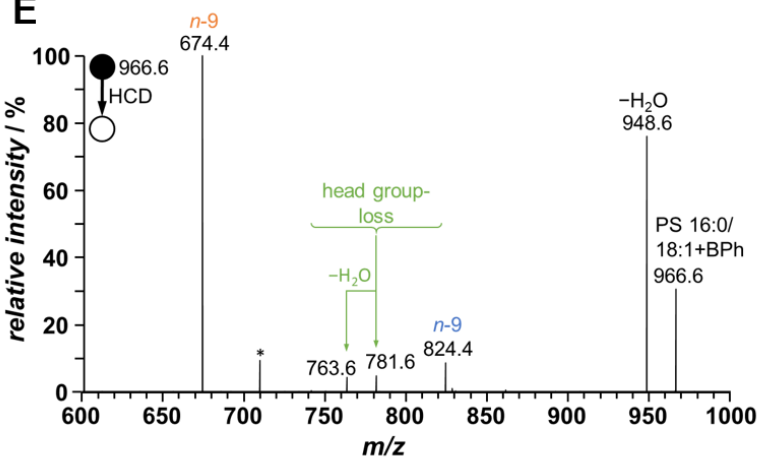

G

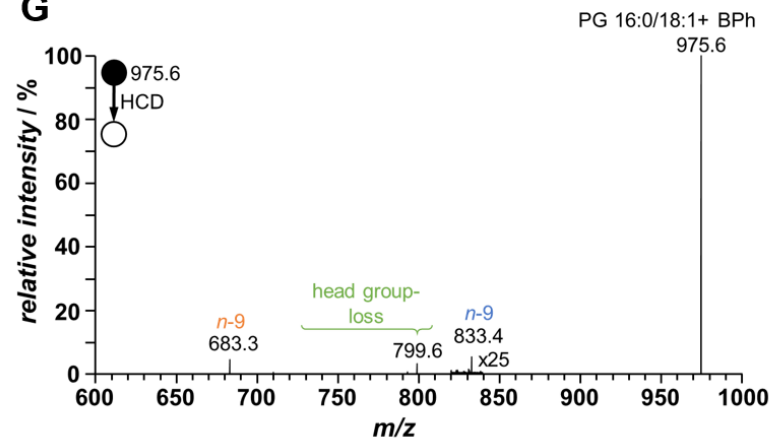

B
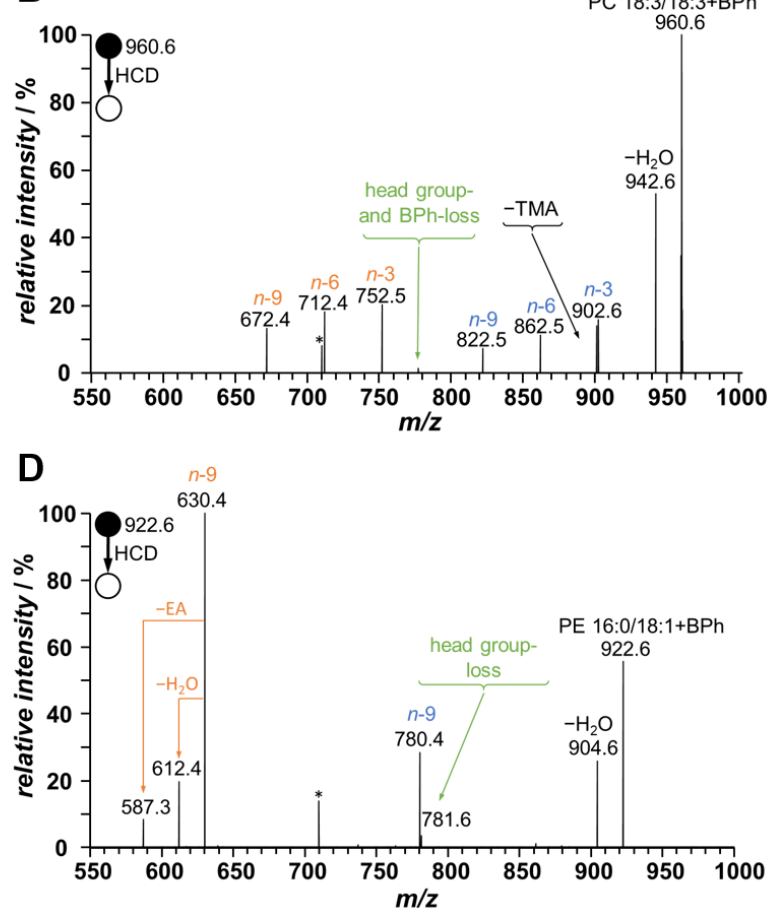

F

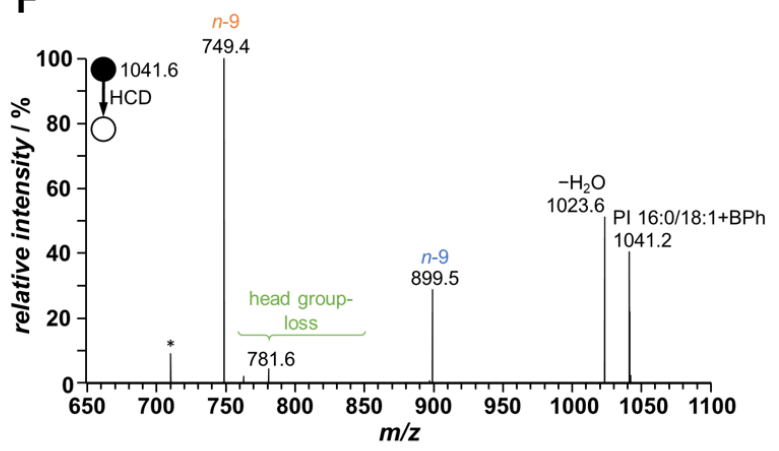

H

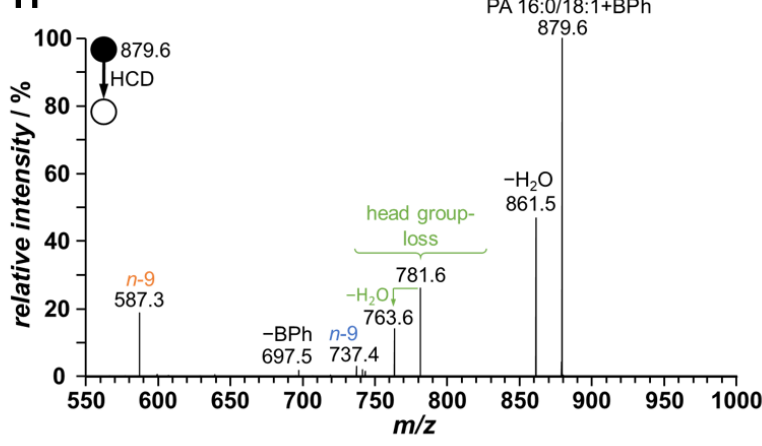

Figure S9. MALDI-HCD-MS ${ }^{2}$-spectra of authentic $\mathrm{PL}$ standards. Indicated PL PB product precursor signals are protonated (A-C and $\mathbf{H}$ ),

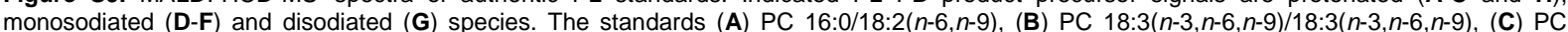
16:0/20:4(n-6,n-9,n-12,n-15), (D) PE 16:0/18:1(n-9), (E) PS 16:0/18:1(n-9), (F) PI 16:0/18:1(n-9), (G) PG 16:0/18:1(n-9) and (H) PA 16:0/18:1(n-9) were used. In all spectra the DB-fragment ion signals are labeled with the respective DB-positions. Aldehyde fragment ion signals are labeled in orange and diphenylethylene fragment ion signals in blue. Signals labeled with a star are electronic noise. Abbreviations: EA - ethanolamine; TMA - trimethylamine. 


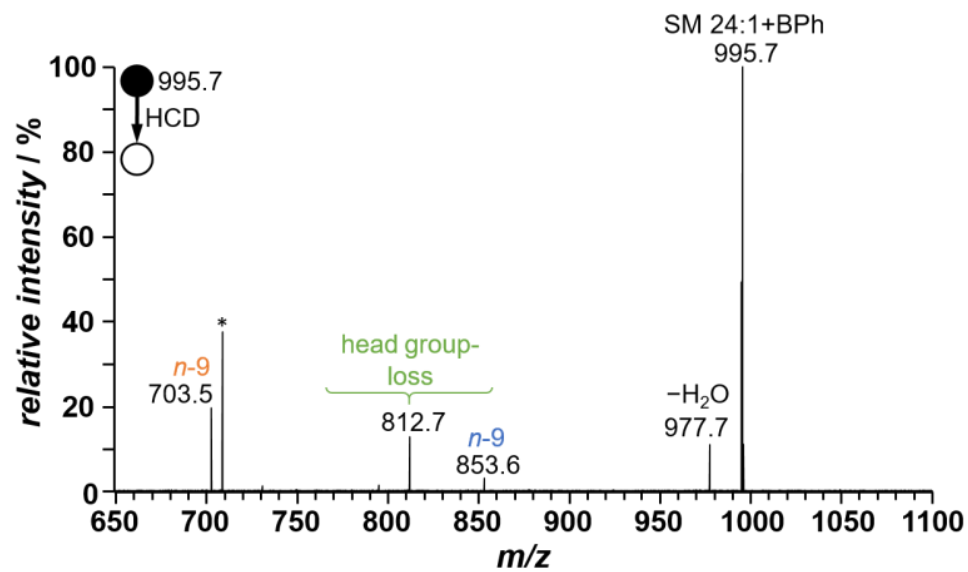

Figure S10. MALDI-HCD-MS ${ }^{2}$-spectrum of protonated PB product ions of SM d18:1(n-14)/24:1(n-9) from SM brain extract. DB-fragment ion signals are labeled with the respective DB positions. Aldehyde fragment ion signals are labeled in orange and diphenylethylene fragment ion signals in blue. Signals labeled with a star are electronic noise.

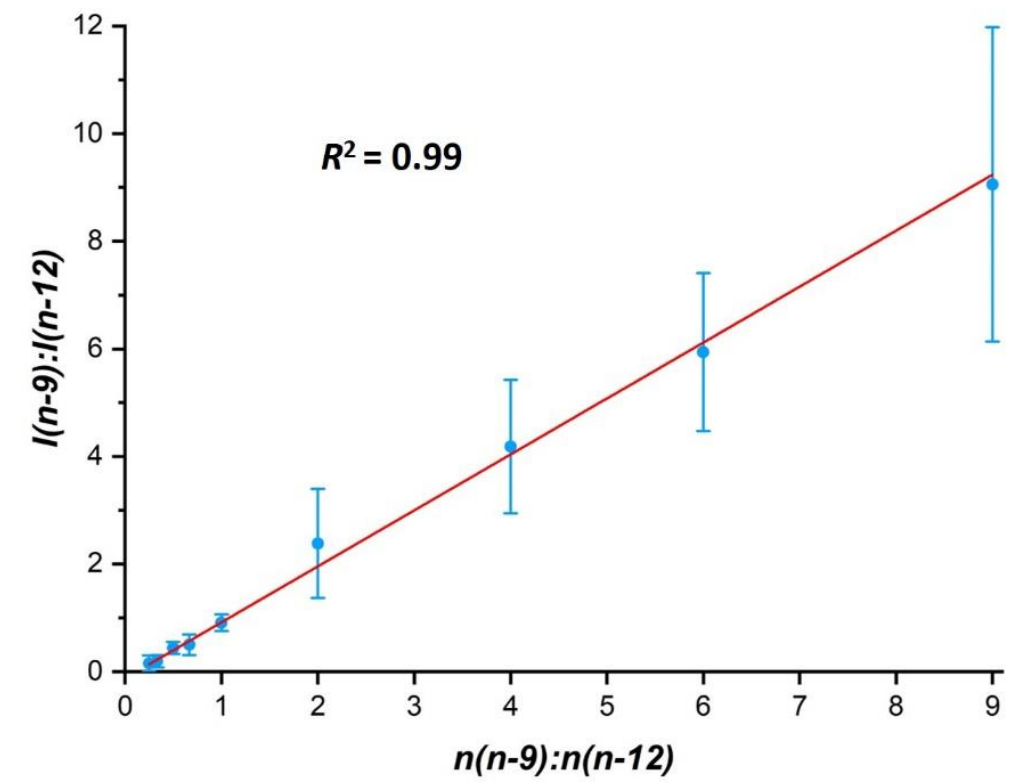

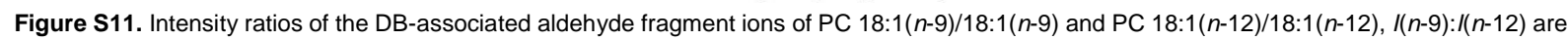
plotted as function of the molar ratios of PC 18:1(n-9)/18:1(n-9) and PC 18:1(n-12)/18:1(n-12), $n(n-9): n(n-12)$. 

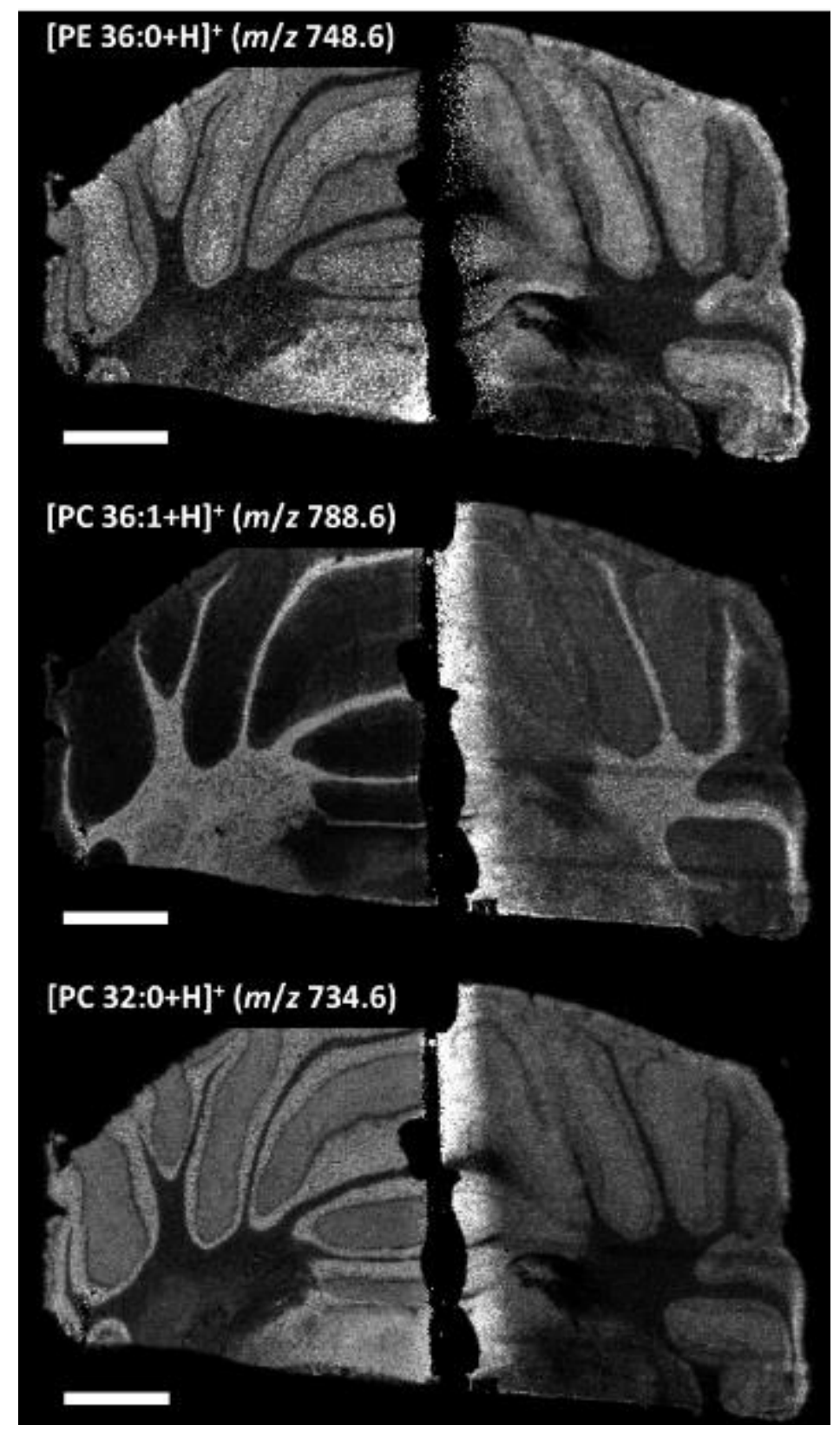

Figure S12. Grayscale MALDI-MS images acquired with BPh (left) and DHB (right) as matrix. The white scale bar reflects $1 \mathrm{~mm}$.
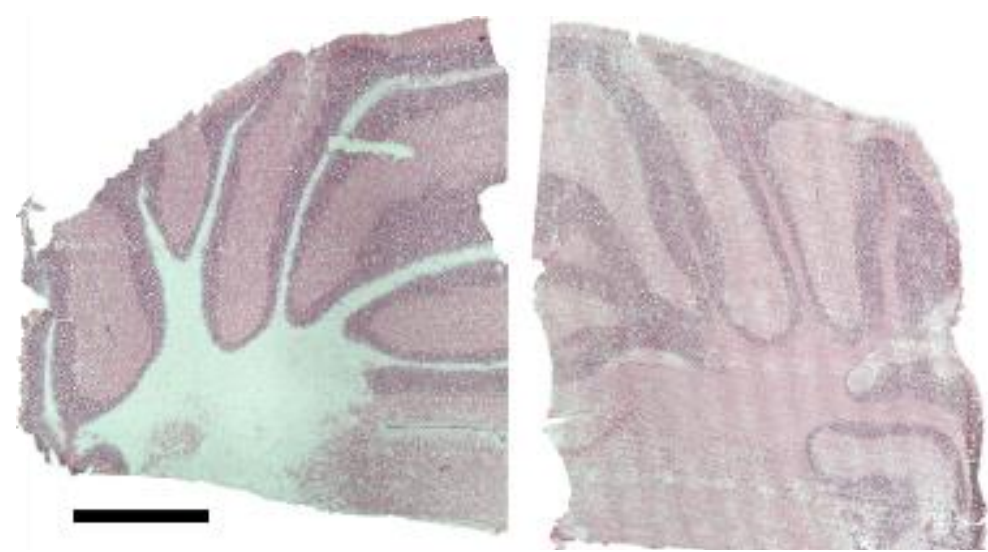

Figure S13. Microscopic images of the two H\&E stained halves of one mouse cerebellum section after MALDI-MS imaging with BPh (left) and DHB (right) as MALDI matrix. The black scale bar reflects $1 \mathrm{~mm}$. 


\section{Benzophenone as MALDI-MSI matrix}

For the evaluation of BPh as MALDI matrix for MALDI-MSI experiments, PL identities and their distributions are compared between (left) BPh and (right) DHB used as matrices in Figure $\mathbf{S 1 2}$ and Table S2. A mouse cerebellum-tissue section was divided in halves, one half was covered with $\mathrm{BPh}$ and the other half with DHB. An example RGB image of resulting PL distributions from MALDI-MSI measurements with $15 \mu \mathrm{m}$ pixel resolution is shown in Figure S14 (grayscale images in Figure S12). The lateral distributions of the three PLs are very similar, irrespective of the matrix compound used. For example when using BPh as matrix, [PC 36:1+H]+ (green) was found to be mainly located in the white matter of brain tissue, consistent with the distribution of the same compound imaged with DHB as matrix. MALDI-MSI results for BPh are also in line with tissue regions identified by optical microscopy (Figure S13). The pixel coverage of $\mathrm{MSI}$ results extracted from tissue region where homogenous PL coverage is expected, e.g. white matter for [PC $36: 1+\mathrm{H}]^{+}$, was typically above $70 \%$, and values for $\mathrm{BPh}$ and DHB were comparable. However, some low-abundant alkali metal PL adducts showed different distributions for BPh compared to DHB. This is most likely a combined effect of ion suppression intrinsic to MALDI and the lower propensity to form PL alkali metal adducts when using BPh compared to DHB. Therefore, BPh used as MALDI matrix generally enables PL MALDI-MSI with resolutions down to $15 \mu \mathrm{m}$ and pixel coverage similar to DHB MALDI-MSI results.

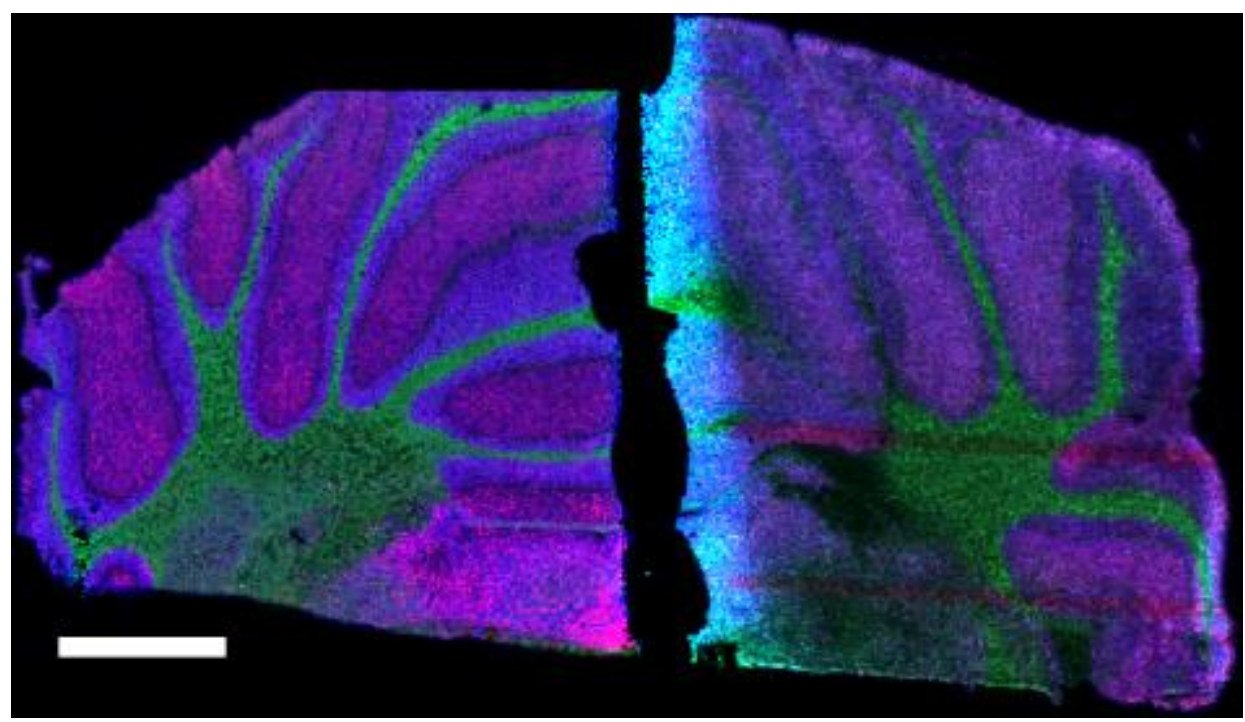

Figure S14. RGB MALDI-MS images acquired with $15-\mu \mathrm{m}$ pixel size using full pixel mode. The lateral resolved signal intensities of three selected lipids were superimposed: $[\mathrm{PE} 36: 0+\mathrm{H}]^{+}(\mathrm{m} / \mathrm{z} 748.585 \text {; red), [PC } 36: 1+\mathrm{H}]^{+}(\mathrm{m} / \mathrm{z} 788.617$; green) and [PC 32:0+H] $(\mathrm{m} / \mathrm{z} 734.570$; blue). Left: BPh; Right: DHB. The white scale bar reflects $1 \mathrm{~mm}$. 

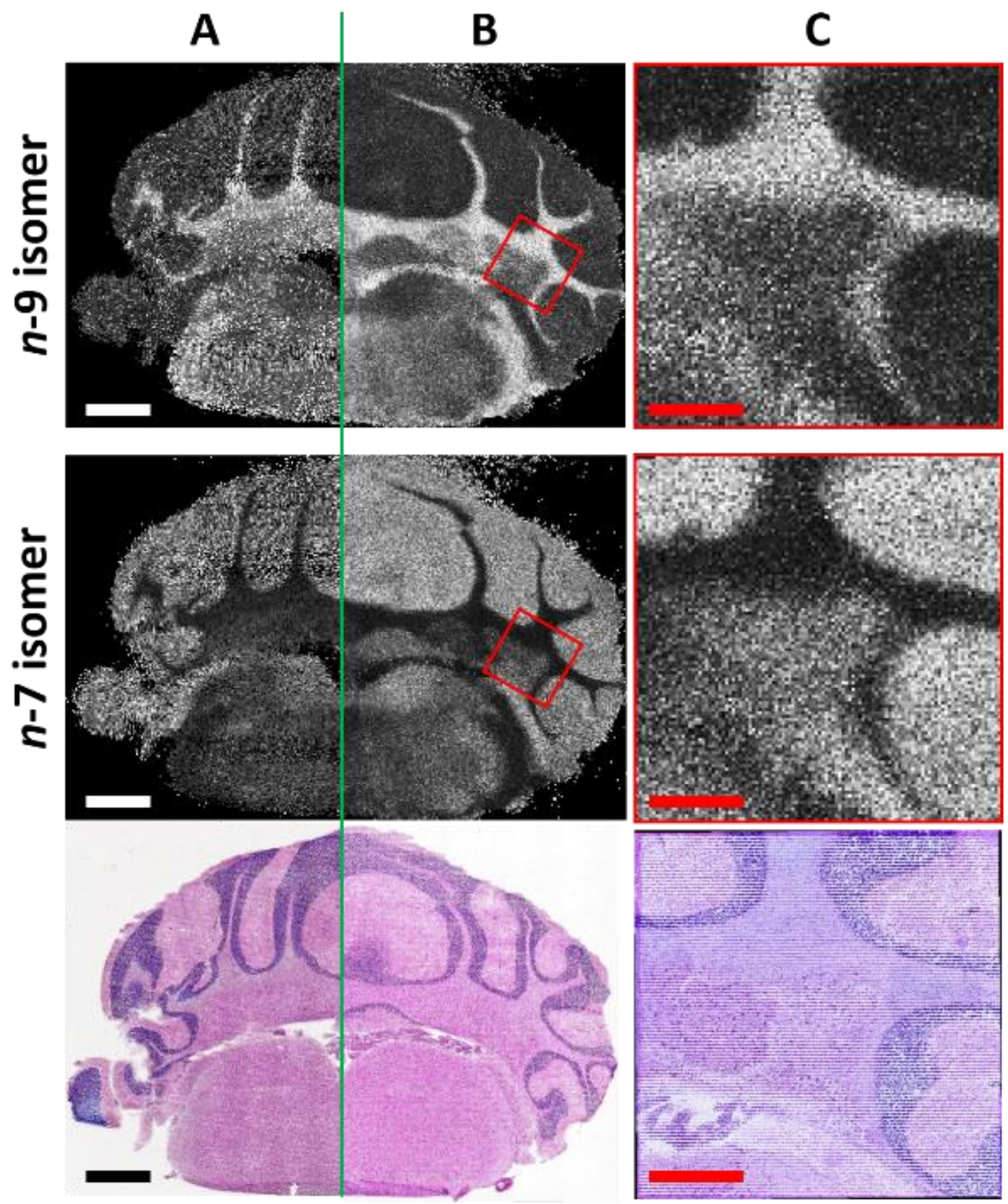

Figure S15. MALDI-MS² images of the diagnostic retro-PB aldehyde fragment ions of (column A) PC $36: 1$ and (columns B,C) PC 34:1, acquired with (A,B) $25 \mu \mathrm{m}$ and (C) $15 \mu \mathrm{m}$ pixel size. The relative intensities of fragment ions associated with (upper row) $n-9 / n-7$ and (middle row) $n-7 / n-9$ are shown. The scale bars in columns A, B and column C reflect $1 \mathrm{~mm}$ and $400 \mu \mathrm{m}$, respectively. The red square in column B highlights the region measured in column C. (lower row) Microscopic images of H\&E stained tissue sections after MALDI-MS²I. 
A
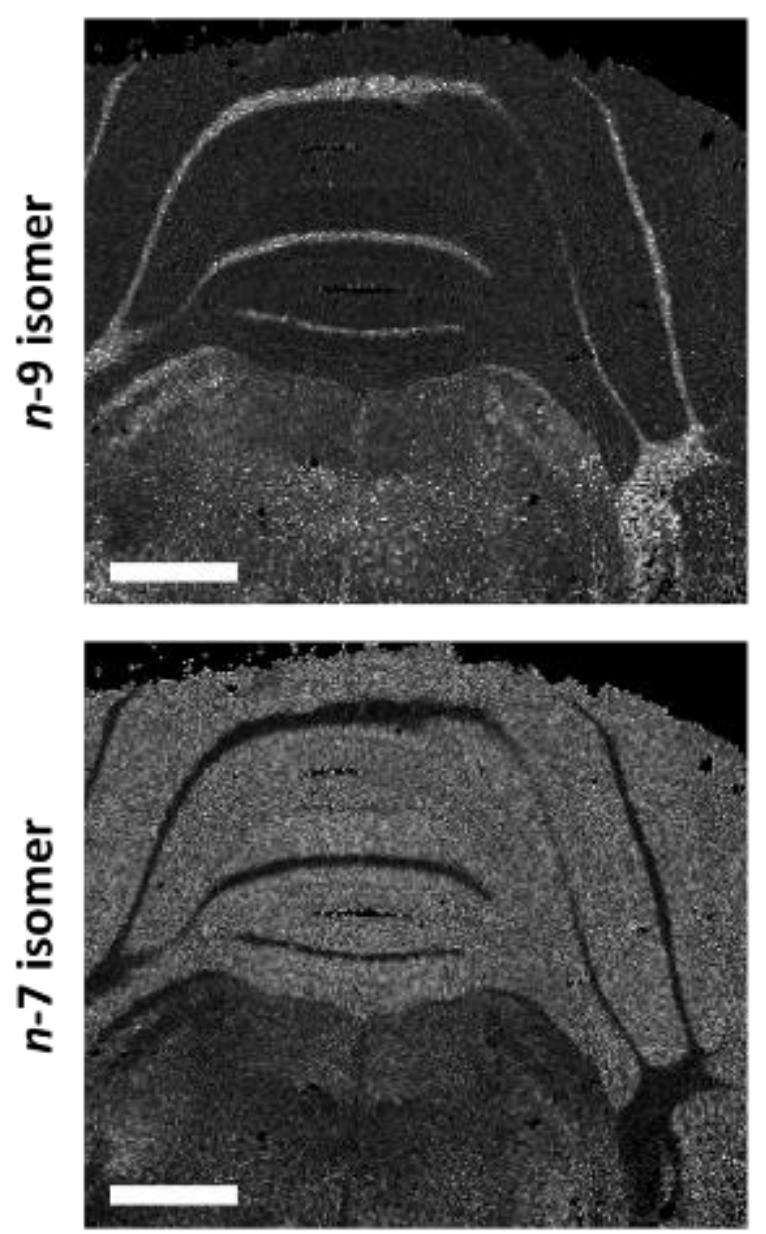

B
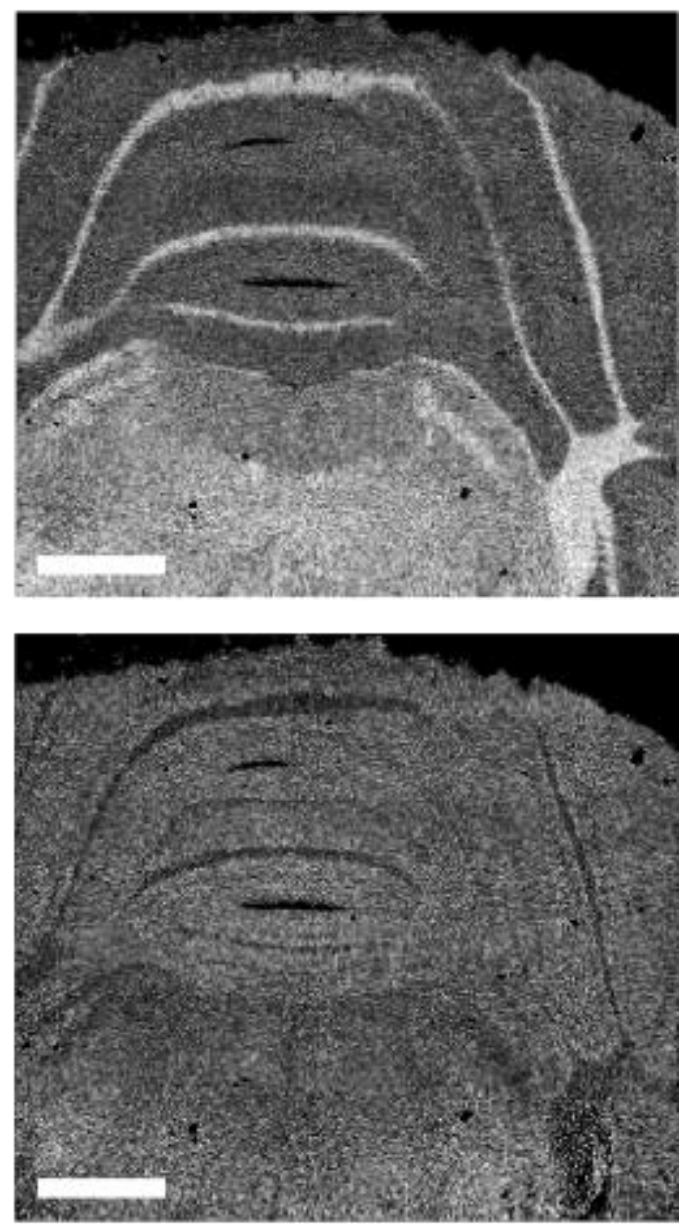

Figure S16. MALDI-MS 2 images of the diagnostic retro-PB aldehyde fragment ions of PC $34: 1$ acquired with $20 \mu \mathrm{m}$ pixel size. (column A) The ion intensities associated with the ratio (upper row) $n-9 / n-7$ and (middle row) $n-7 / n-9$ are shown as a measure for relative isomer abundance. (column B) TIC-normalized lateral distributions of (upper row) the $n-9$ and (middle row) the $n-7$ DB-position isomer. The scale bars reflect 1 mm. (lower row) Microscopic image of the H\&E stained tissue section after MALDI-MS²I. 

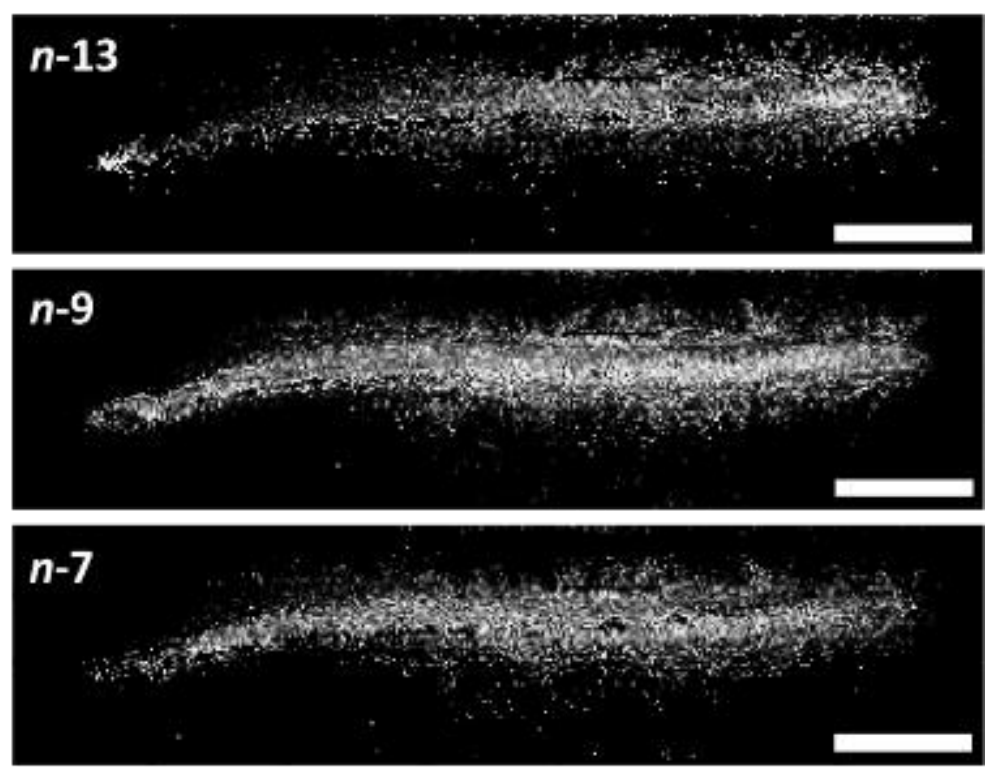

\section{Optical image}

Figure S17. (upper three rows) MALDI-MS ${ }^{2}$ images of diagnostic retro-PB aldehyde fragment ions of PC $34: 1$ acquired from male $S$. mansoni tegument using full pixel mode and employing triangulation height correction with $20 \mu \mathrm{m}$ pixel size. This is a biological replicate of the $S$. mansoni worm analyzed in Figure 3. Ion intensities were TIC normalized. (lower row) Microscopic image before measurement. The scale bars reflect $1 \mathrm{~mm}$.
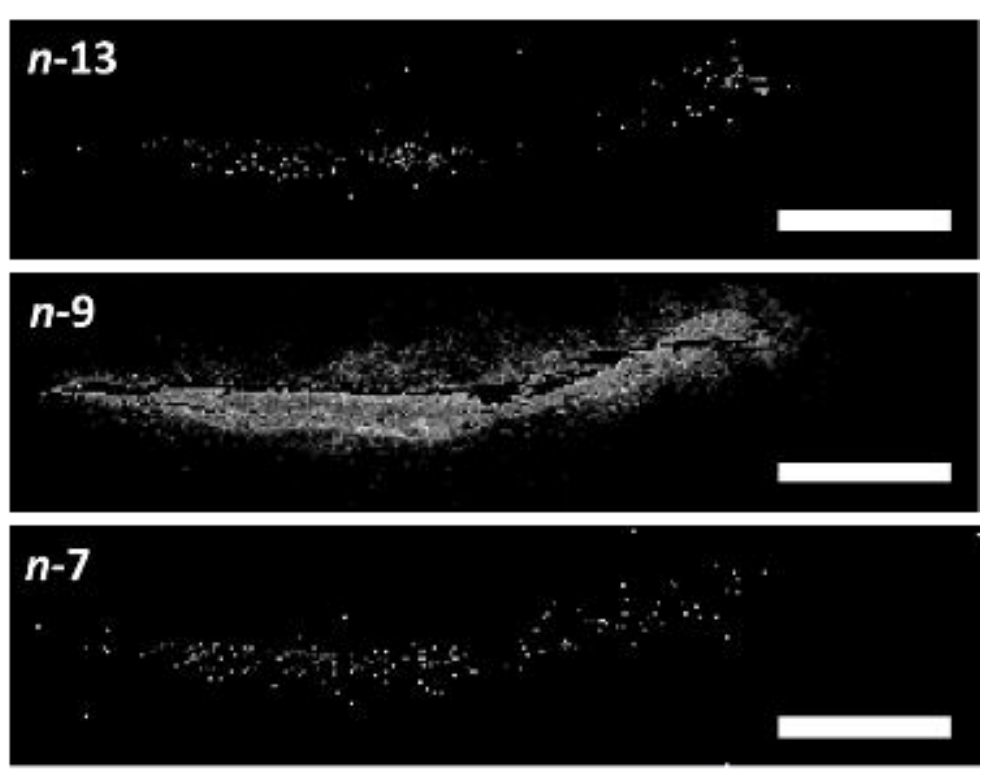

\section{Optical image}

Figure S18. (upper three rows) MALDI-MS ${ }^{2}$ images of diagnostic retro-PB aldehyde fragment ions of PC $36: 1$ acquired from male $S$. manson tegument using full pixel mode and employing triangulation height correction with $20 \mu \mathrm{m}$ pixel size. The ion intensities are TIC normalized. (lower row) Microscopic image before measurement. The scale bars reflect $1 \mathrm{~mm}$. 

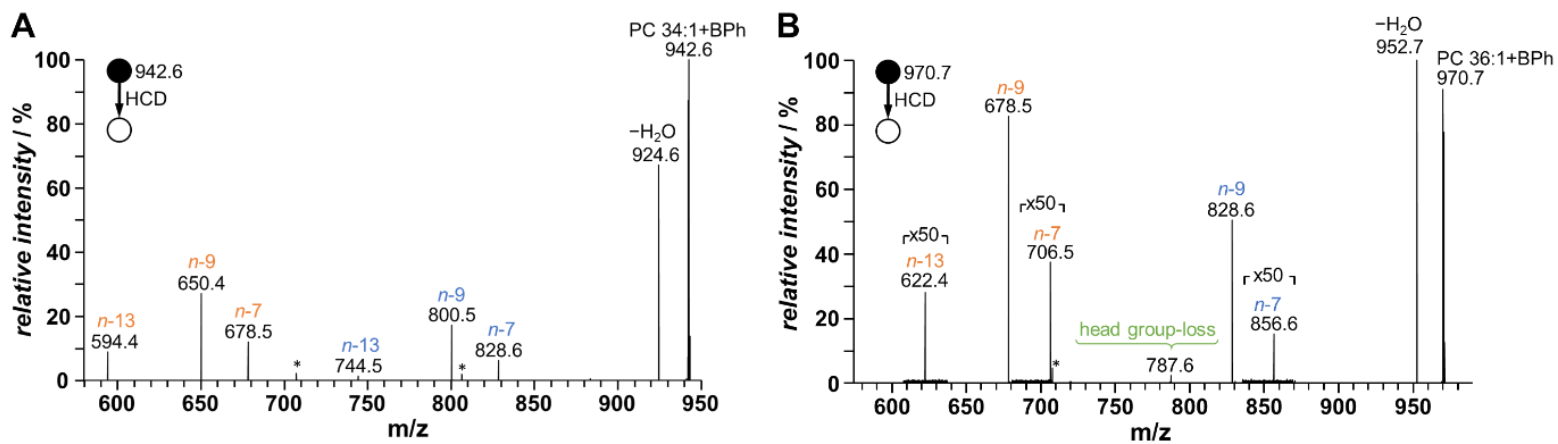

Figure S19. MALDI-HCD-MS²-spectrum of protonated PB product ions of (A) PC 34:1 and (B) PC $36: 1$ from male S. mansoni tissue. DB-fragment ion signals are labeled with the respective DB-positions. Aldehyde fragment ion signals are labeled in orange and diphenylethylene fragment ion signals in blue. The signals labeled with a star are electronic noise.

A

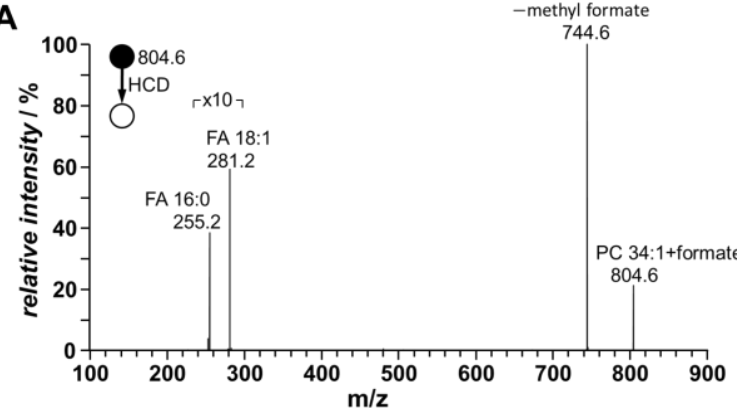

B

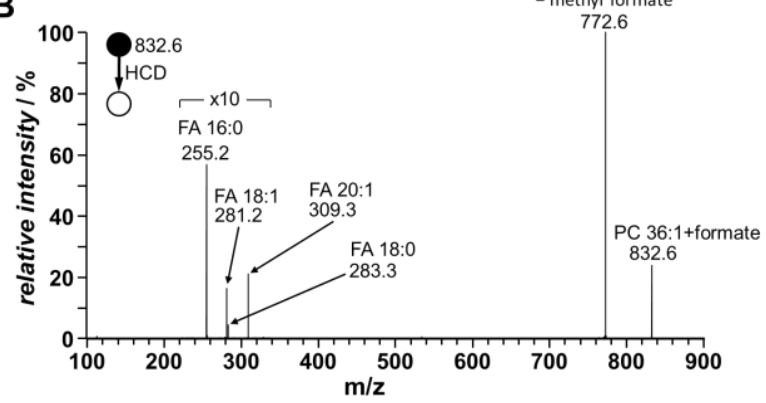

Figure S20. Negative ion mode nanoESI-HCD-MS²-spectrum of format adducts of (A) PC 34:1 and (B) PC 36:1 from male S. mansonitissue extract. FA ion signals are labeled with the respective chain length and degree of unsaturation.
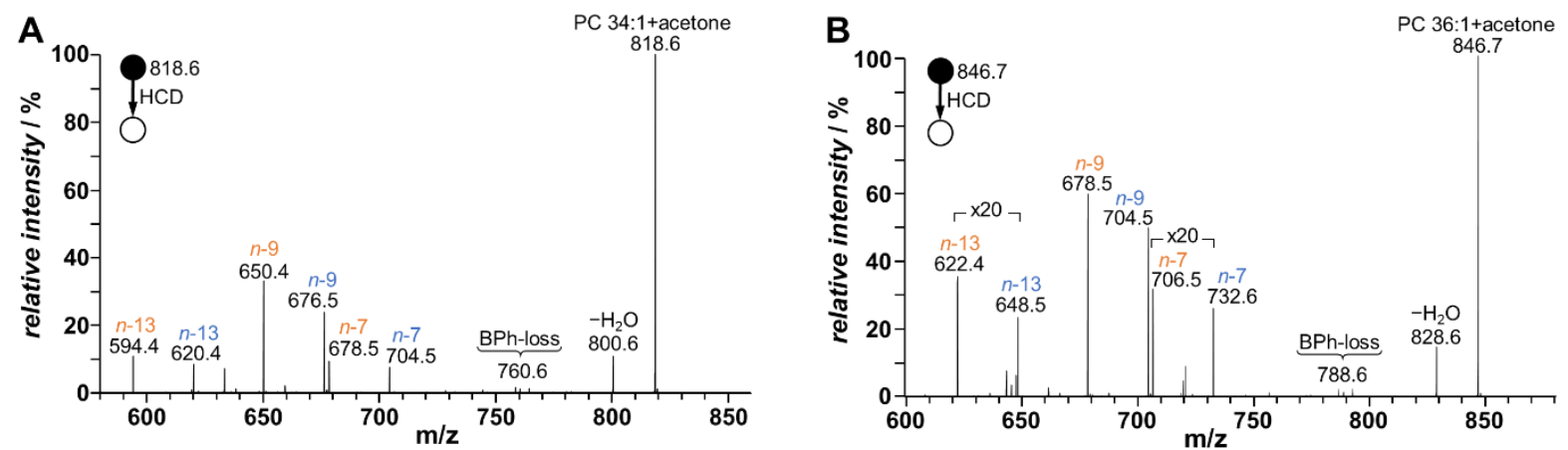

Figure S21. Positive ion mode nanoESI-HCD-MS²-spectrum of protonated PB product ions of acetone and (A) PC $34: 1$ and (B) PC $36: 1$ from male $S$. mansoni tissue extract. DB-fragment ion signals are labeled with the respective DB-positions. Aldehyde fragment ions are labeled in orange and dimethylethylene fragment ions in blue. Fragment ions below $1 \%$ relative intensity were omitted. 


\section{Supporting Protocol 1 - Pneumatic Spraying Optimization}

Taking into account that the PB reaction takes place during the MALDI process, the microextraction of lipid molecules in the BPh matrix crystals had to be maximized to enhance the reaction yield and the total analyte intensity. Besides the micro-extraction improvement, the matrix crystal size was a crucial optimization objective because small crystal sizes are required for high-resolution MALDI-MSI. Therefore, following parameters were optimized: concentration of BPh (5-90 mg/mL) and trifluoroacetic acid (0.2-0.5\%), solvent composition, deposited BPh amount $(100-400 \mu \mathrm{L})$, pumping flow rate $(5-20 \mu \mathrm{L} / \mathrm{min})$ and pneumatic gas flow. Matrix spraying-solutions have to be highly concentrated to allow homogeneous crystallization on tissue after solvent evaporation. Although, the concentration has to be low enough to prevent clogging of the sprayer capillary. For the solvent composition, two properties were essential: the ability to dissolve BPh and the vapor pressure of the resulting matrix solvent-mixture to ensure fast crystallization without analyte migration due to solvent transport. Acetonitrile, methanol, ethanol, 2-propanol and water were tested in various compositions. After optimization, acetonitrile was used as solvent capable to dissolve BPh and the analyzed lipids. 2-Propanol and water were utilized because of their lower vapor pressure in comparison to acetonitrile to adjust the volatility of the solvent mixture. Pumping flow rate and pneumatic gas flow were adjusted to obtain complete tissue sample coverage and small crystal sizes (see

Figure S2A). Based on these criteria the optimized spray protocol was: $230 \mu \mathrm{L}$ of the spraying solution containing $20 \mathrm{mg} / \mathrm{mL}$ BPh in acetonitrile:2-propanol:water 6:3:1 complemented by $0.5 \%$ trifluoroacetic acid were sprayed with $10 \mu \mathrm{L} / \mathrm{min}$.

\section{Supporting Protocol 2}

1) Dehydration in ethanol and set to demineralized water
1.1) $100 \%$ ethanol
$2 \min$

1.2) $70 \%$ ethanol

$2 \min$

1.3) $40 \%$ ethanol

$2 \min$

1.4) Demineralized water

$2 \min$

2) Hematoxylin

$12 \min$

3) Washing in tap water

$10 \min$ 
4) $1 \%$ aqueous Eosin solution

$1 \min$

5) Differentiation in ethanol

5.1) Demineralized water

$2 \min$

5.2) $40 \%$ ethanol

$2 \min$

5.3) $70 \%$ ethanol

$2 \min$

5.4) $100 \%$ ethanol

6) Xylol

7) Eukitt@ fixation

\section{References}

(1) Liebisch, G.; Vizcaino, J. A.; Kofeler, H.; Trotzmuller, M.; Griffiths, W. J.; Schmitz, G.; Spener, F.; Wakelam, M. J. O. Shorthand notation for lipid structures derived from mass spectrometry. J. Lipid Res. 2013, 54, 1523-1530.

(2) IUPAC-IUB Commission on Biochemical Nomenclature. The nomenclature of lipids. Lipids 1977, 12, 455-468.

(3) Grevelding, C. G. The female-specific W1 sequence of the Puerto Rican strain of Schistosoma mansoni occurs in both genders of a Liberian strain. Mol. Biochem. Parisitol. 1995, 71, 269-272.

(4) Hankin, J. A.; Barkley, R. M.; Murphy, R. C. Sublimation as a method of matrix application for mass spectrometric imaging. J. Am. Soc. Mass Spectrom. 2007, 18, 1646-1652.

(5) Kompauer, M.; Heiles, S.; Spengler, B. Atmospheric pressure MALDI mass spectrometry imaging of tissues and cells at 1.4- $\mu$ m lateral resolution. Nat. Methods 2017, 14, 90-96.

(6) Kompauer, M.; Heiles, S.; Spengler, B. Autofocusing MALDI mass spectrometry imaging of tissue sections and 3D chemical topography of nonflat surfaces. Nat. Methods 2017, 14, 1156-1158.

(7) Robichaud, G.; Garrard, K. P.; Barry, J. A.; Muddiman, D. C. MSiReader: an open-source interface to view and analyze high resolving power MS imaging files on Matlab platform. J. Mass Spectrom. 2013, 24, 718-721.

(8) Matyash, V.; Liebisch, G.; Kurzchalia, T. V.; Shevchenko, A.; Schwudke, D. Lipid extraction by methyl-tert-butyl ether for highthroughput lipidomics. J. Lipid Res. 2008, 49, 1137-1146.

(9) Hu, C.; van Dommelen, J.; van der Heijden, R.; Spijksma, G.; Reijmers, T. H.; Wang, M.; Slee, E.; Lu, X.; Xu, G.; van der Greef, J.; Hankemeier, T. RPLC-ion-trap-FTMS method for lipid profiling of plasma: method validation and application to p53 mutant mouse model. J. Proteome Res. 2008, 7, 4982-4991.

(10) Esch, P.; Heiles, S. Charging and Charge Switching of Unsaturated Lipids and Apolar Compounds Using Paternò-Büchi Reactions. J. Am. Soc. Mass Spectrom. 2018, 29, 1971-1980. 